\title{
Genome-wide identification of candidate aquaporins involved in water accumulation of pomegranate outer seed coat
}

\author{
Jianjian Liu ${ }^{\text {Equal first author, } 1,2}$, Gaihua Qin ${ }^{\text {Equal first author, } 2,3}{ }^{3}$ Chunyan Liu ${ }^{2,3}$, Xiuli Liu ${ }^{4}$, Jie Zhou ${ }^{4}$, Jiyu Li ${ }^{2,3}$, Bingxin Lu ${ }^{1}$, \\ Jianrong Zhao ${ }^{\text {Corresp. } 1}$ \\ ${ }^{1}$ College of Resource and Environment, Anhui Science and Technology University, Fengyang, China \\ 2 Institute of Horticultural Research(Key Laboratory of Genetic Improvement and Ecophysiology of Horticultural Crop, Anhui Province), Anhui Academy of \\ Agricultural Sciences, Hefei, China \\ 3 Key Laboratory of Fruit Quality and Developmental Biology, Anhui Academy of Agricultural Sciences, Hefei, China \\ 4 State Key Laboratory for Managing Biotic and Chemical Threats to the Quality and Safety of Agro-products, Zhejiang Academy of Agricultural Sciences, \\ Hangzhou, China \\ Corresponding Author: Jianrong Zhao \\ Email address: zhaojr@ahstu.edu.cn
}

Aquaporins (AQPs) are a class of highly conserved integral membrane proteins that facilitate the uptake and transport of water and other small molecules across cell membranes. However, little is known about AQP genes in pomegranate (Punica granatum L.) and their potential role in water accumulation of the outer seed coat. We identified 38 PgrAQP genes in the pomegranate genome and divided them into five subfamilies based on a comparative analysis. Purifying selection played a role in the evolution of PgrAQP genes and a whole-genome duplication event in Myrtales may have contributed to the expansion of PgrTIP, PgrSIP, and PgrXIP genes. Transcriptome data analysis revealed that the PgrAQP genes exhibited different tissue-specific expression patterns. Among them, the transcript abundance of PgrPIPs were significantly higher than that of other subfamilies. The mRNA transcription levels of PgrPIP1.3, PgrPIP2.8, and PgrSIP1.2 showed a significant linear relationship with water accumulation in seed coats, indicating that PgrPIP1.3/PgrPIP2.8 located in the plasma membrane and PgrSIP1.2 proteins located on the tonoplast may be involved in water accumulation and contribute to the cell expansion of the outer seed coat, which then develops into juicy edible flesh. Overall, our results provided not only information on the characteristics and evolution of PgrAQPs, but also insights on the genetic improvement of outer seed coats. 
1 Title: Genome-wide identification of candidate aquaporins involved in water

2 accumulation of pomegranate outer seed coat

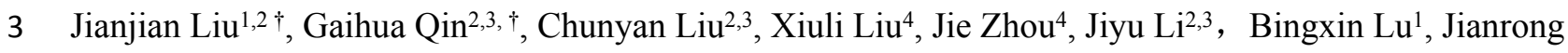
$4 \mathrm{Zhao}^{1, *}$

5 1. College of Resource and Environment, Anhui Science and Technology University, Fengyang, China.

6 2. Institute of Horticultural Research, Anhui Academy of Agricultural Sciences (Key Laboratory of

7 Genetic Improvement and Ecophysiology of Horticultural Crop, Anhui Province), Hefei, China.

8 3. Key Laboratory of Fruit Quality and Developmental Biology, Anhui Academy of Agricultural Sciences, 9 Hefei, China.

4. State Key Laboratory for Managing Biotic and Chemical Threats to the Quality and Safety of Agroproducts, Zhejiang Academy of Agricultural Sciences, Hangzhou, China.

$\dagger$ These authors have contributed equally to this work and share first authorship.

${ }^{*}$ Corresponding author:

Jianrong Zhao, College of Resource and Environment, Anhui Science and Technology University, Fengyang, China.

E-mail: zhaojr@ahstu.edu.cn

\section{Abstract}

Aquaporins (AQPs) are a class of highly conserved integral membrane proteins that

facilitate the uptake and transport of water and other small molecules across cell membranes. However, little is known about AQP genes in pomegranate (Punica granatum L.) and their potential role in water accumulation of the outer seed coat. We 
23 identified 38 PgrAQP genes in the pomegranate genome and divided them into five

24 subfamilies based on a comparative analysis. Purifying selection played a role in the

25 evolution of $\operatorname{Pgr} A Q P$ genes and a whole-genome duplication event in Myrtales may

26 have contributed to the expansion of PgrTIP, PgrSIP, and PgrXIP genes. Transcriptome

27 data analysis revealed that the $P g r A Q P$ genes exhibited different tissue-specific

expression patterns. Among them, the transcript abundance of PgrPIPs were

significantly higher than that of other subfamilies. The mRNA transcription levels of

PgrPIP1.3, PgrPIP2.8, and PgrSIP1.2 showed a significant linear relationship with water

accumulation in seed coats, indicating that PgrPIP1.3/PgrPIP2.8 located in the plasma

membrane and PgrSIP1.2 proteins located on the tonoplast may be involved in water

accumulation and contribute to the cell expansion of the outer seed coat, which then

develops into juicy edible flesh. Overall, our results provided not only information on

the characteristics and evolution of $P g r A Q P s$, but also insights on the genetic

improvement of outer seed coats.

\section{Introduction}

Pomegranate (Punica granatum L.) is an important economic fruit tree species due

to its functional and nutraceutical properties, and it is widely consumed as a fruit, juice, 
44 Spain, Egypt, Iran, Morocco, the USA, China, India, Argentina, Israel, and South Africa

45 (Qin et al. 2017). The size of the juicy outer seed coat determines the edible quality of

46 the fruit, so it has become an important fruit characteristic. Notably, the morphological

47 characteristics of the pomegranate seed showed a compressed inner seed coat and an

48 expanded outer seed coat, making it an appealing model for studying development of seed coats (Luo et al. 2020; Niu et al. 2018; Qin et al. 2020).

The development of the seed coat is accompanied by the import of organic and inorganic nutrients, including sugars, organic acids, cellulose, and water in developing seeds (Qin et al. 2020; Uçar \& Karagöz 2009; Zarei et al. 2016; ZHOU et al. 2007b). The symplastic pathway is one of the main pathways by which water moves, which is mediated by integral membrane proteins called aquaporins (AQPs), a major intrinsic protein family (Adams \& Wendel 2005; Lian et al. 2004; Suga et al. 2002). AQPs have been shown to transport small molecules such as carbon dioxide, glycerol, ammonia, urea, hydrogen peroxide, and selenite (Ivanov et al. 2007; Yasui et al. 1999; Zwiazek et al. 2017a).

The general AQP structure is highly conserved in plants, and it is predicted to consist of six transmembrane (TM) $\alpha$-helices (H1 to H6) and two half-helices (Deshmukh et al. 2015; Lee et al. 2005; Tornroth-Horsefield et al. 2006). These transmembrane $\alpha$-helices are linked by five short loops (Loops A to E), Loops B and E contain the signature sequence Asparagine-Proline-Alanine (NPA) motif, which has the primary function of forming water-selective channels (Chaumont et al. 2001; Gupta \& 
65 Sankararamakrishnan 2009; Wallace \& Roberts 2004). Another important secondary

66

67 structure known as an aromatic/arginine $(\mathrm{ar} / \mathrm{R})$ selectivity filter is formed by four residues contributed by transmembrane helices $\mathrm{H} 2 / \mathrm{H} 5$ and the loop LE (Azad et al. 2016; Deshmukh et al. 2015; Hove \& Bhave 2011; Tornroth-Horsefield et al. 2006). These two structures predominantly determine the specificity of solute transport and transport rate (Deshmukh et al. 2015; Lee et al. 2005; Tornroth-Horsefield et al. 2006). According to sequence similarity and protein subcellular localization, AQPs in higher plant can be classified into five distinct subfamilies: plasma membrane intrinsic proteins (PIPs), tonoplast intrinsic proteins (TIPs), nodulin 26-like intrinsic proteins (NIPs) (Pommerrenig et al. 2015), small basic intrinsic proteins (SIPs), and unrecognized (or X) intrinsic proteins (XIPs)(Chaumont et al. 2001; Danielson \& Johanson 2010; Kaldenhoff \& Fischer 2006; Khabudaev et al. 2014; Quigley et al. 2002).

The AQP gene family has been widely studied in numerous plant species, such as Arabidopsis thaliana (Quigley et al. 2002), Oryza sativa (Sakurai et al. 2005), Glycine max (Zhang et al. 2013), cotton (Li et al. 2019), Populus trichocarpa (Gupta \& Sankararamakrishnan 2009), and grape (Fouquet et al. 2008), by blasting whole genome sequences. Previous studies have demonstrated that AQPs in higher plants play important roles in various physiological and development processes, such as cell and tissue expansion, fiber development, flower pollination, and seed development (Azad et al. 2004; Eisenbarth \& Weig 2005; Gattolin et al. 2011; Soto et al. 2008; Soto et al. 2010; Vander Willigen et al. 2006; Wudick et al. 2014). For instance, in French bean (Phaseolus 
86

87

vulgaris), PvPIPs played a role in the import of water and nutrients by phloem-mediated transport and water recycling in the xylem in developing seeds (Terashima \& Ono 2002). In rice, OsPIP1;1 and OsPIP1;3 functions as water channels. Over-expression of OsPIP1;1 could increase rice yield and seed germination. Similarly, the overexpression of OsPIP1;3 promoted the speed of seed germination under drought conditions (Liu et al. 2013; Liu et al. 2007). In Pisum sativum L., PsNIP1 showed high expression level in developing pea seed coats, and the overexpression of PsNIP1 increased the uptake of water and glycerol (Schuurmans et al. 2003; Zhou et al. 2007a). Interestingly, the highly correlations between the expression of AtTIP3/AtTIP1 and seed germination stages (a rapid imbibition of desiccated tissues and embryo growth, respectively) was observed, which also provided insight into the influence of AQPs on the seed developmental process (Gattolin et al. 2011). However, little is known about the role of AQPs on seed coat development.

In this study, we identified $38 \operatorname{Pg} r A Q P$ genes in the pomegranate genome, then conducted gene structure, phylogenetics, and evolutionary divergence analyses. The expression patterns of $\operatorname{Pg} A Q P$ genes in different tissues and seed developmental stages were analyzed, and the potential function of PgrPIP genes in response to drought stress was also identified. Furthermore, the candidate genes contributing to the water accumulation in the seed coat were selected based on a correlation analysis of gene expression and water accumulation in seed coats. Our findings provided fundamental information about the gene structure, phylogenetics, and evolutionary divergence of 
107 PgrAQP. In addition, this study also provided useful information for further

108 investigation of the molecular mechanism by which PgrAQP functions in seed coat

109 development.

110 2. Materials and Methods

111

113

114

115

116

117

118

119

120

121

122

123

124

125

126

\subsection{Identification of the PgrAQP genes in the pomegranate genome}

The whole genome sequences of pomegranate were downloaded from NCBI

Genome (https://www.ncbi.nlm.nih.gov/genome/?term=Punica+granatum+L). Predicted protein sequences were obtained using TBtools with the following sets: Sequence

Toolkit: Batch translate CDS to protein (Chen et al. 2020). The amino acid sequences of the AQPs in Arabidopsis and Populus trichocarpa were employed as queries to blast searches against the whole-genome sequences in pomegranate using TBtools with a query over $50 \%$ and e-value less than 0.01 (Quigley et al. 2002). The candidate sequences were submitted to NCBI for EST blast searches. Finally, the AQP protein sequences of pomegranate were examined to verify the presence of the characteristic MIP and transmembrane helical domains using the SMART program (http://smart.embl-heidelberg.de/) and TMHMM (http://www.cbs.dtu.dk/services/TMHMM/) (Finn et al. 2014; Letunic et al. 2020). The information of PgrAQP gene family (protein length, molecular weight, and isoelectric point) were determined using Expasy (https://web.expasy.org/compute_pi/) (Artimo et al. 2012). The subcellular localization of the PgrAQP proteins was predicted by WoLF 
127 PSORT (http://www.genscript.com/psort/wolf_psort.html) Cell-PLoc 2.0

128 (http://www.csbio.sjtu.edu.cn/bioinf/Cell-PLoc-2/)

129

130

131

132

133

134

135

136

137

138

139

140

141

142

143

144

145

146

147

2.2 Sequence alignment of PgrAQP genes, phylogenetic analysis, and classification

The predicted plant AQP protein sequences were aligned using the ClustalW tool

in MEGA7.0 (Kumar et al. 2016). Arabidopsis thaliana, grape (Vitis vinifera), Populus

trichocarpa and eucalyptus (Eucalyptus grandis) AQP protein sequences were collected

from NCBI (https://www.ncbi.nlm.nih.gov/). A phylogenic tree was constructed with

MEGA7.0 using the neighbor-joining method and bootstrap parameter with 1,000

replicates. For this study, Pomegranate AQPs were named according to the sequence

homology and phylogenetic relationships with Populus trichocarpa. According to the

name of the best hit gene in Populus trichocarpa, the subfamily classification (PIP, NIP,

SIP, TIP, and XIP) and corresponding names of AQPs are shown in Fig. 1. TBtools

software was used to determine the localization of $\operatorname{Pgr} A Q P$ genes on pomegranate

chromosomes.

\subsection{Gene structure and conserved motif analysis of PgrAQPs}

The conserved motifs in the proteins were identified using Multiple Expectation

Maximization for Motif Elucidation (MEME v4.12.0, http://meme-suite.org/tools/meme)

with the following parameters: maximum number of motifs, 10; width of optimum

motif, $\geq 15$ and $\leq 50$ (Bailey et al. 2009). The gene exon-intron structures of AQPs were

confirmed using the GSDS database by aligning the open reading frame (ORF) with

their corresponding genomic sequences (Hu et al. 2015a). Sequences representing NPA 
148 motifs, ar/R filter, and Froger's residue positions were manually identified based on

149 multiple sequence alignments of pomegranate AQPs with heterologous AQPs of

150 Arabidopsis (Kumar et al. 2016). TBtools software was used to construct a chromosome

151 localization map of AQP family genes.

152 2.4 Gene duplication and synteny analysis of the PgrAQP gene family

153 Gene duplication events of PgrAQP genes were analyzed using TBtools with the

154 following parameters: Blast Compare Two Seqs, Outfml: Table, NumofThreads: 2, E-

155 value:1e-5, NumofHits: 5, NumofAligns: 5; File Merger For MCScanX, Merge Mode:

156 GtfGff2 SimGxf (Chen et al. 2020). Duplication types were divided into whole-genome

157 (WGD), segmental, and tandem duplications (Qiao et al. 2015). Tandem duplicated

158 genes were defined as two homologous genes within a range of 100-kb and sequence

159 alignment coverage over 75\% (Gu et al. 2002; Wang et al. 2010; Yang et al. 2008). The

160 nonsynonymous substitution ratios $(K a)$, synonymous substitution ratios $(K s)$, and

$161 \mathrm{Ka} / \mathrm{Ks}$ ratios of the PgrAQP family genes were calculated using the TBtools simple $\mathrm{Ka} / \mathrm{Ks}$

162 calculator program, with the following parameters: Simple Ka/Ks Calculator (NG). The

163 Ks value was used to calculate the divergence time of duplication events ( $T=K s / 2 R$ Mya,

164 Millions of years), where $\mathrm{R}$ is the rate of synonymous substitutions, $\mathrm{R}=1.5 \times 10^{-8}$

165 substitutions per synonymous site per year for dicotyledonous plants and T refers to

166 divergence time (Edlund et al. 2004). The microsyntenic relationship of AQP genes

167 between pomegranate, grape, Arabidopsis, and eucalyptus was determined using

168 TBtools Dual Systeny Plot for MCscanX program (Chen et al. 2020). 
169

170

171

172

173

174

175

176

177

178

179

180

181

182

183

184

185

186

187

188

189

\subsection{Plant materials and analysis of transcript profiles of PgrAQP genes}

Two major pomegranate cultivars, 'Dabenzi' (a hard-seeded cultivar) and 'Tunisia' (a soft-seeded cultivar), were planted in Anhui Province (Hefei, 31 $51^{\prime} 9.05^{\prime \prime} \mathrm{N}$,

$\left.117^{\circ} 06^{\prime} 34.33^{\prime \prime} \mathrm{E}\right)$, China and grown under the same fertilization and irrigation conditions.

Seeds from five fruits of the pomegranate cultivar 'Dabenzi' and 'Tunisia' were

collected at 50, 95, and 140 days after pollination (DAP). For outer seed coats, the seeds

from fruits collected 95 DAP and 140 DAP was used because it was difficult to visually

distinguish the inner and outer seed coats of seeds were collected at 50 DAP. Three

biological replicates were taken for RNA sequencing. For each treatment, the fresh

weights of the total inner and outer seed coats were recorded and immediately frozen in

liquid nitrogen. Part of the inner and outer seed coats were stored at $-80^{\circ} \mathrm{C}$ until they

were used for transcriptome sequencing. RNA extraction was performed as described

previously (Qin et al. 2020). Briefly, the total RNA was isolated using a Plant RNeasy

Mini Elute Cleanup kit (Qiagen, Beijing, China) and the sequencing libraries were

prepared using the NEBNext Ultra ${ }^{\mathrm{TM}}$ RNA Library Prep Kit for Illumina (New England

Biolabs, USA) following the manufacturer's protocols. Transcriptome sequencing was

conducted using an Illumina HiSeq 2000 platform.

The remaining samples were used for the measurement of water accumulation. The water accumulation (\%) in the different seed coats was determined by the fresh weight $(\mathrm{FW})$ and drought weight $(\mathrm{DW})$ using the formula $(\mathrm{FW}-\mathrm{DW}) / \mathrm{FW} \times 100$. The freeze-

drying analysis was performed using GOLD-SIM FD83 (SIM International group co. Itd, 
190

191 192 193

194 195 196 197 198 199 200 201

USA). The experiments were repeated three times.

The abundances of pomegranate AQP transcripts, in the root, flower, leaf, and three developmental stages of the peel and seed coat (inner and outer seed coat), were collected from the microarray data previously published by Qin et al., (Qin et al. 2017). Transcriptional abundances of AQP genes were estimated using the fragments per kilobase of exon per million mapped reads (FRKM) method, and a heat map was generated based on the $\log _{2}$ FRKM transformation value using TBtools (Hu et al. 2018), with the following protocol: Starting from the "Graphics", click "Heatmap lllustrator", select the Heatmap button, then set input files in each filed, click "Start" and graph will be generated.

\subsection{Verification of PgrAQP genes function responding to water deficit in}

\section{pomegranate root}

For the analysis of PgrAQP genes expression patterns in response to water deficit, the PEG treatment experiment was performed. For the hydroponic culture experiment, the full Hoagland nutrient solution contained 20\% (v/v) polyethylene glycol 6000 (PEG6000). The solution $\mathrm{pH}$ was adjusted to 5.5. The roots samples were collected after

$0 \mathrm{~h}, 1 \mathrm{~h}, 6 \mathrm{~h}, 12 \mathrm{~h}$ and $24 \mathrm{~h}$ of treatment, and were quickly frozen in liquid nitrogen and stored at -80 for subsequent RNA isolation.

For performing qRT-PCR analysis, approximately 2 ug of NDA-eliminated total RNA from pomegranate roots were used to synthesize cDNA using a reverse transcription kit (TaKaRa). qRT-PCR was performed on the Applied Biosystems (ABI) 
211 StepOne Plus PCR system (Applied Biosystems) using the LightCylcer 96 SYB GREEN I

212 Master (Roche, Indianapolis, IN, USA) in a 20 ul reaction solution. The PgrAQP genes

213 that showed higher expressed in the roots from the RNA-Seq data were selected for

214 qRT-PCR analysis. The relative transcript abundance of each gene was normalized to

215 the pomegranate actin (OWM91407) with the cycle threshold (Ct) $2\left({ }^{-} \Delta \Delta \mathrm{Ct}\right)$ method. Three

216 biological replicates and technical replicates were used for each gene. All the specific

217 primers used for each target gene are listed in Table. S1.

218 2.7 Statistical analysis

219 The data were analyzed by ANOVA (SPSS 16.0; SPSS Inc., Chicago, IL, USA),

220 followed by Turkey's test $(\mathrm{P}<0.05)$ to determine differences of inner and outer seed coats.

221 The data represent the mean \pm SE of three independent biological replicates.

222 3. Results

223 3.1 Genome-wide identification of PgrAQP genes in pomegranate reveal each

224 Aquaporin subfamily presents particular physicochemical characteristics

225 A total of 38 PgrAQP members were identified in the pomegranate genome via a

226 genome-wide search using the AQP protein sequences in Arabidopsis and Populus

227 trichocarpa as queries (Quigley et al. 2002). A subsequent conserved domain analysis

228 also confirmed all of the predicted AQPs (Fig. S1). The characteristics of PgrAQP family

229 genes are shown in Table. S2, including the gene ID, protein length, relative molecular

230 weight, transmembrane domains (TMDs), subcellular localization, and calculated

isoelectric point $(\mathrm{pI})$. The 38 predicted PgrAQP genes encoded proteins that varied in 
232 length from 245 to 359 amino acid residues, with a relative molecular weight of 22.86 to

$23335.18 \mathrm{kDa}$, and a calculated pI ranging from 5.06 to 10.16. The average PI value of

234 PgrTIPs was less than other AQPs due to the loss of basic residues in the C-terminal

235 domain. The grand average of hydrophobicity index (GRAVY) was used to evaluated

236 protein hydrophobicity and hydrophilicity. The results showed the GRAVY of PgrAQP

237 proteins were all positive, ranging from 0.203 to 0.967 , which indicated that all of

238 PgrAQP proteins were hydrophobic. Furthermore, the lowest average of GRAVY value

239 (0.44) was found in the PgrPIP subfamily, suggesting that this subfamily has better

240 interaction with water molecules.

241 The predicted transmembrane domains (TMDs) showed that most PgrAQP genes

$242(28$ of 38, 73.7\%) contained six TMDs, 2.7\%, 15.8\%, and 7.8\% of PgrAQP genes contained

243 four, five, and seven TMDs, respectively (Fig. S2). Based on subcellular localization

244 predicted by WoLF PSORT, most PgrPIP and PgrNIP proteins were predicted to

245 localize in plasma membranes, while only PgrPIP2.2 was found in the chloroplast. All

246 PgrTIP proteins were predicted to localized to vacuoles. For PgrSIP proteins, PgrSIP1.1

247 and PgrSIP1.2 were found in the chloroplast and vacuole, respectively. PgrXIP proteins

248 were predicted to localized in plasma membranes.

2493.2 Phylogenetic characterization of the pomegranate PgrAQP gene family

250 To investigate the evolutionary relationship of pomegranate AQP family genes, a

251 total of 193 AQP protein sequences from the four species studied (38 in pomegranate, 35

252 in Arabidopsis, 33 in grape, 55 in Populus trichocarpa and 40 in eucalyptus) were 
253 identified. An unrooted phylogenetic tree was constructed based on the alignments of

254 their amino acid sequences in MEGA 7 using the neighbor-joining method (Kumar et al.

255 2016). By comparing amino acid sequences of PgrAQPs with APQs from three other

256 plant species, 38 PgrAQPs were divided into five different subfamilies, that is, 14

257 PgrTIPs, 13 PgrPIPs, eight PgrNIPs, two PgrSIPs, and one PgrXIP (Fig. 1). The PgrPIPs

258 divided into two major subgroups, PgrPIP1s and PgrPIP2s, which comprised five and

259 eight members, respectively. Furthermore, PgrNIPs formed six subgroups (PgrNIP1,

260 PgrNIP1, PgrNIP4, PgrNIP5, PgrNIP7, and PgrNIP8) in pomegranate. As the largest sub-

261 family, the TIPs members were classified into five subgroups, consisting of seven

262 PgTIP1s, two PgTIP2s, two PgTIP3s, one PgTIP4, and two PgTIP5s. SIPs and XIPs

263 formed one group, containing two and one members, respectively. In the phylogenetic

264 tree, PgrAQP genes were more closely related to AQPs in eucalyptus than that in

265 Arabidopsis and grape, which is in accordance with the evolutionary relationships

266 among these species (Qin et al. 2017).

267

268

269

270

271

272

273

\subsection{Gene structure and conserved motif analysis of PgrAQP genes confirm the} phylogenetic classification

Gene structure and conserved motifs can provide information for exploring the evolutionary relationships among a gene family. The number of introns of PgrAQP genes ranged from zero to four, while the length of exons was highly similar for each subfamily (Fig. S3). Among them, most of the PgrTIPs genes had two introns, except for PgrTIP1.6 and PgrTIP1.8, which had only one intron. For PgrPIP genes, most of 
274 members had three introns, whereas PgPIP2.8 contained two introns. The numbers of

275 introns in PgrNIPs ranged from one to four introns. Four out of 10 members had four

276 introns (PgrNIP2.1, PgrNIP4.1, PgrNIP4.2, and PgrNIP3.1), three members had three

277 introns (PgrNIP1.2, PgrNIP5.1, and PgrNIP7.1), and two members had two introns

278 (PgrNIP1.1 and PgrNIP1.3). PgrXIP2.1 had three introns. The SIP family formed a small

279 subfamily, among which, two members had two introns and one member had no

280 introns.

281 By analyzing the intron-exon structure of PgrAQP genes, we found that the

282 number of introns of each AQPs subfamily (PIP, NIP, TIP, SIP, and XIP) are highly

283 conserved and similar when compared with plant species, such as banana, watermelon,

284 chickpea, and sweet orange, suggesting similar intron loss or gain events were

285 experienced in AQP subfamily over the course of evolution (Deokar \& Tar'an 2016; Hu

286 et al. 2015b; Martins Cde et al. 2015; Zhou et al. 2019). Multiple sequence alignments

287 showed that PgrNIPs and PgrPIPs were the most diverse (38.5\%) and conserved (72.7\%)

288 subfamily at the amino acid level, respectively (Table. S3). This finding is consistent

289 with the AQPs from Nicotiana tabacum, suggesting that the function and regulatory

290 mechanism of PgrPIP subfamily genes showed more conserved than PgrNIP subfamily

291 genes in pomegranate (De Rosa et al. 2020; Deokar \& Tar'an 2016). Furthermore, the

292 relative conservation of protein sequences and ar/R selectivity filter among the PgrPIP

293 and PgrTIP subfamily genes suggested that these proteins may share a conserved

294 function in transporting water and other small neutral solutes (Table. S3 and S4) (Zhu 
295

296

297

298

299

300

301

302

303

304

305

306

307

308

309

310

311

312

313

314

315

et al. 2019).

To detect the structural diversity and provide further support of the grouping of PgrAQPs, a total of 10 conserved motifs were identified (Fig. S3). Generally, motif compositions were conserved within each subfamily. For PgrPIP, eight motifs were found in all family members. Motifs $1,2,3,6,8$, and 10 were common for TIP and NIP subfamily members, except for PgrNIP7.1. Interestingly, motifs 4 and 5 were only identified in the PIP subfamily and similar motifs were found in bread wheat, indicating the PIP subfamily may have unique functions (Madrid-Espinoza et al. 2018).

\subsection{Comparison of substrate-specific residues in PgrAQP proteins}

The NPA motifs, ar/R selectively filter, and Froger's positions were identified by multiple sequence alignment between the PgrAQPs and AtAQPs using MEGA 7.0.

These highly conserved motifs and positions were critical for the substrate selectively of AQPs (Tornroth-Horsefield et al. 2006). Conserved domain searches using CDD tool from NCBI confirmed all the predicted AQP genes in Pomegranate encoded MIP domains (Fig.S7). As shown in Table. S4, all of PgrTIPs, PIPs, and XIP proteins harbored two conserved NPA domains in both loop B (LB) and loop E (LE). For the PgrNIPs subfamily, except for PgrNIP5.1, the rest of numbers showed the third residue of the first NPA motifs was serine rather than alanine. In addition, PgrSIP1.2 showed the substitution of alanine by threonine in their first NPA motif.

14 The residues of the ar/R selectivity filter and Froger's position displayed conserved regions within each subfamily, but regions were more variable across different 
subfamilies (Kayum et al. 2017). For example, all of the members of the PgrPIPs showed conservative residues at the ar/R selectively filter with phenylalanine-histidinethreonine-arginine, which is typical of aquaporin protein structure. The residues of Froger's position were conserved in PgrPIPs, including S at P2, A at P3, Y at P4, and W at P5, while the P1 position was variable with the Q/M residues. Different residues were observed at the ar/R selectively filter and Froger's position in PgrNIPs, which had W/G/A/V-V/S/I-A/G-R and F/L/Y-S/T-A-Y/F-I/V/LM residue compositions, respectively.

For PgrTIPs members, the residues of P3, P4, and P5 positions were highly conserved, while the P1 and P2 positions were variable residues. The ar/R selectivity filters were variable with $\mathrm{H} / \mathrm{S} / \mathrm{N}-\mathrm{I} / \mathrm{V}-\mathrm{A} / \mathrm{P}-\mathrm{V} / \mathrm{R} / \mathrm{C}$ residues. The residues of Froger's position were conserved in PgrSIP1.1 and PgrSIP1.2, but the ar/R filter showed distinct difference. specificity (Almasalmeh et al. 2014; Hove \& Bhave 2011). The highly conserved amino acid features of AQPs included six transmembrane domains, the NPA domain, and the ar/R selectivity filter(Froger et al. 1998). For example, all of the PgrPIP subfamily members showed a highly conserved ar/R filter structure (T-H-R-T) (Table. S4), which was observed in PIP family genes from other plant species, such as watermelon, Arabidopsis, Brassica rapa, soybean, and chickpea, indicating that the substrate specificity of this subfamily may be more specific than others subfamilies (Deokar \& Tar'an 2016; 
337 observed. Furthermore, the highly conserved ar/R filter (H-I-A-V) and Froger's

338 positions (T-S-A-Y-W) of the PgrTIP1 subfamily were reported to function as urea and

$339 \mathrm{H}_{2} \mathrm{O}_{2}$ transporter (Hove \& Bhave 2011). In addition, the conserved ar/R filter G-S-G-R

340 residues were found in PgrNIP2.1, and this characteristic was identified as the indicator

341 of Si transporters, indicating that PgrNIP2.1 may be involved in the transport of Si

342 (Deshmukh et al. 2013; Deshmukh et al. 2015; Zhou et al. 2019). In pomegranate, valine

343 was present at position H5 in the ar/R selectivity filters of PgrXIP2.1, suggesting that the

344 hydrophobicity of PgrXIP2.1 is greater than other PgrAQPs subfamily members

345 (Danielson \& Johanson 2008; Gupta \& Sankararamakrishnan 2009).

3.5 Segmental duplication events have contributed to the expansion of the PgrAQP

347

348

349

350

351

352

353

354

355

356

357

\section{family genes}

To investigate the localization of PgrAQP genes and duplication events in pomegranate, we anchored the PgrAQPs on chromosomes and conducted a duplication analysis. The physical position of the PgrAQP genes were found to be unevenly

distributed across all pomegranate chromosomes (Fig. 2). The PIP subfamily genes were randomly anchored on chromosomes, except for Chromosome 7, and TIP subfamily genes were found in all chromosomes except Chromosome 4. Aside from Chromosomes 2 and 8, NIP group genes were located in each chromosome. Genes in the SIP subfamily were present only on Chromosome 4.

We further analyzed the gene duplication modes of PgrAQP genes in pomegranate. As shown in Fig. 2, 47\% of the PgrAQP genes had been duplicated by

Peer) reviewing PDF | (2020:12:56609:2:0:NEW 8 Jun 2021) 
358

359

360

361

362

363

364

365

366

367

368

369

370

371

372

373

374

375

376

377

378

tandem/segmental duplication events. We found one tandem duplication event in

Chromosome 3 (PgrTIP1.2/PgrTIP1.7). There were eight pairs of segmental duplications detected among six chromosomes. As shown in Table 1, the proportion of segmental PgrAQP gene duplications was $88 \%$, indicating that segmental duplication events have played a key role in the expansion of the PgrAQP gene family. To access the selection pressure and the date at which such duplication events occurred, estimation of the Ka and Ks substitution rates of these duplication $\operatorname{Pgr} A Q P$ gene pairs were calculated. A Ka/Ks ratio $>1$ and $<1$ indicate positive Darwinian selection or purifying selection, whereas a value of 1 indicates neutral selection. The Ka/Ks ratios of PgrAQP duplication gene pairs showed a $K a / K s$ ratio of $<1$, indicating that these $\operatorname{Pgr} A Q P$ genes have experienced purifying selection during the course of evolution. According to the mathematical formula $\left(\mathrm{T}=K_{s} / 2 \lambda\right)$ used to calculate the evolutionary date, we assessed the divergence time of these duplication events and found that the gene duplication events occurred approximately 1.74-6.97 million years ago.

Further, we constructed the comparative synteny maps of three plants species (pomegranate vs. Arabidopsis, pomegranate vs. grape, and pomegranate vs. Eucalyptus) to explore the evolutionary process of PgrAQP genes (Fig. 3, Table. S5) and found that 24, 28, and 41 orthologous AQP gene pairs were identified, respectively. Remarkably, the numbers of orthologous TIP gene pairs between pomegranate and grape/Eucalyptus were significantly higher than that in Arabidopsis. Nevertheless, the PIP genes were highly conserved within the species. The syntenic relationship detected in TIP genes 
379 indicates that the expansion of PgrTIPs and VvTIPs/EucTIPs genes may have occurred

380 after that of Arabidopsis, while the PIP genes have been evolutionarily conserved.

3.6 Analysis of PgrAQP gene expression profiles of different pomegranate tissues

and water deficit

Identifying tissue-specific genes is a basic strategy to select candidate genes involved in

biological processes. To explore the possible functions of PgrAQP genes in various

developmental stages of different organs of pomegranate, a heat map of PgrAQP

expression profiles was conducted. The heat map showed various expression patterns

of the 38 PgrAQP genes analyzed (Fig. 4A, Table. S6). Most of TIPs, such as PgrTIP1.1,

PgrTIP1.4, PgrTIP1.6, PgrTIP1.8, and PgrTIP2.1, showed higher expression in the roots,

leaves, and flowers, whereas the transcripts of PgrTIP2.3, PgrTIP3.1, and PgrTIP3.2

showed extremely low expression levels. For the NIPs subfamily, PgrNIP4.1 and

PgrNIP5.1 were highly expressed in the leaves and roots, respectively. Interestingly, the

transcripts of PgrNIP1.3 could only be detected in flowers, indicating that PgrNIP1

might be involved in the development of pomegranate flowers, while other members

were transcribed at extremely low levels. The accumulation of transcripts of two

PgrSIPs was detected in all tissues analyzed, whereas PgrSIP1.2 had higher relative

expression levels than that of PgrSIP1.1. In the PIPs subfamily, PgrPIP3, PgrPIP2.3, and

PgrPIP2.4 had a low level of transcripts in all tissues and stages analyzed, whereas the

remaining members had higher expression levels in all tissues analyzed. Notably, 
400

401

402

403

404

405

406

407

408

409

410

411

412

413

414

415

416

417

418

419

420

and seed coats at all three experimental stages, indicating that these genes may play important roles in seed development.

To reveal the potential function of PgrAQPs in response to water deficit in pomegranate root, the transcript levels of seven selected PgrAQPs from PIP subfamily for seedling under 20\% PEG supply condition were tested using qRT-PCR. According to the qRT-PCR results, except the PgrPIP1.5, of which the transcript was enhanced in the roots after the $24 \mathrm{~h}$ of $20 \%$ PEG supply conditions, the expression of the other five paralogues, PgrPIP1.1, PgrPIP1.2, PgrPIP1.3, PgrPIP2.1 and PgrPIP1.4, were significantly repressed under the 20\% PEG supply condition (Figure 5). It should be noted that the expression levels of the PgrPIP1.2 was strongly and rapidly decreased under the 20\% PEG treatment. In contrast, the expression pattern of PgrPIP2.4 showed barely changed until $12 \mathrm{~h}$ after 20\% PEG treatment, the down-regulation was observed at $24 \mathrm{~h}$.

\subsection{Identification of candidate PgrAQPs involved in water accumulation in the outer} seed coat of pomegranate

To understand water transport and accumulation in pomegranate seed coats, especially of the juicy outer seed coat, we detected water accumulation in the inner and outer seed coats at different developmental stages, as well as the relative transcript levels of PgrAQP genes in corresponding samples. We found that the water content was significantly increased in outer seed coats during seed development in 'Dabenzi' and 'Tunisia,' and the water that accumulated in the outer seed coats was higher than that in 
421 the inner seed coats (Fig. 6). Genes, including PgrPIP1.3, PgrPIP2.8, PgrPIP1.5,

422 PgrPIP2.6, PgrPIP2.1, PgrPIP2.2, PgrPIP2.5, and PgSIP1.2, had relatively high levels of

423 transcript accumulation in inner and outer seed coats. Among the abovementioned

424 genes, PgrPIP1.3, PgrPIP2.8, and PgSIP1.2, had high levels of accumulation of

425 transcripts only in the outer seed coat at the later developmental stages (Fig. 5), which

426 indicated that these genes may be involved in the accumulation of water in outer seed

427 coats at the later developmental stages.

428 In Arabidopsis, a plasma membrane aquaporin, AtPIP1;2 is involved in aquaporin-

429 mediated leaf water transport, lateral root formation, and water uptake in root. To

430 further understand the function of PgrPIP1.3, PgrPIP2.8, and PgrSIP1.2 in the water

431 accumulation in the seed coat, the linear relationship between the water content in the

432 seed coat and differences in transcript levels for PrgPIP1.3 and PgrSIP1.2 in seed coat

433 development was determined. Significant positive correlations in both PgrPIP1.3 and

434 PgrSIP1.2 and water content in outer seed coats were observed (Fig. 7). Therefore, it was

435 concluded that PgrPIP1.3 and PgrSIP1.2 might be involved in the accumulation of water

436 in pomegranate seed coats.

437 4. Discussion

$438 \quad 4.1$ PgrAQP identification and structure analysis

439 AQP proteins play an important role in various physiological and developmental

440 processes of different organs and tissues, and function as a transporter for water and/or

441 small neutral solutes in plants. Numerous studies have been conducted on AQPs in 
442 plants, such as in Arabidopsis, grape, soybean, rice, and Populus trichocarpa (Fouquet et al.

443 2008; Gupta \& Sankararamakrishnan 2009; Quigley et al. 2002; Sakurai et al. 2005;

444 Zhang et al. 2013). However, the genome-wide identification of the AQP gene family in

445 pomegranate was absent due to the limitations of an available genome sequence (Luo et

446 al. 2020; Qin et al. 2017). In this study, 38 PgrAQP genes were identified and

447 characterized in pomegranate. The characteristics of PgrAQP were comparable with

448 other plants species. For instance, the numbers of exons and structures of intron/exons

449 in the PgrAQP genes were highly conserved in different species, such as Arabidopsis,

450 olive, chickpea, Arachis hypogea, and banana (Deokar \& Tar'an 2016; Faize et al. 2020; Hu

451 et al. 2015b; Quigley et al. 2002; Shivaraj et al. 2019). Similar to the organizations of

452 intron/exons, PgrAQP proteins of each subfamily possess the same conserved motifs

453 (Fig. 2). These results suggest that the gene structures of PgrAQPs are closely related to

454 homologous genes.

455 Furthermore, the TMDs showed that some of the PgrAQPs were lack one

456 (PgrSIP1.1) or two (PgrNIP5.1, PgrPIP2.8) TMDs (Fig. S3). Variation in the number of

457 TMDs to different plant species has been reported (Ayadi et al. 2011; Zhu et al. 2019).

458 For instance, a truncated form of wheat TdPIP2;1 aquaporin, showed no water transport

459 activity. Interestingly, the truncated tdpip2;1 could reach the plasma membrane by

460 interact with the functional TdPIP2;1, and then may affect the functional form and

461 reduce the water transport activity of aquaporin (Ayadi et al. 2011). Therefore, the

462 absence of TMDs may affect the PgrAQPs subcellular localization and water transport 
463 activity.

464 4.2 The evolutionary relationships among the PgrAQP gene family

465 Gene duplication is considered as a major driving force for the evolution of gene

466 families, and several duplication events have been identified over the course of

467 evolution of some plant species. At approximately 117 million years ago (Mya), all core

468 eudicots experienced a genome triplication event (the $\gamma$ event), including Arabidopsis,

469 Eucalyptus, grape, and pomegranate (Jiao et al. 2012; Jiao et al. 2014). Then, Arabidopsis

470 experienced two recent WGDs ( $\alpha$ and $\beta$ ), whereas pomegranate and Eucalyptus

471 underwent a Myrtales lineage-specific WGD event (109.9Mya, M), but grape did not

472 undergo any additional WGDs (Myburg et al. 2014; Qin et al. 2017). In Arabidopsis, a

473 total of 35 AQP genes were identified and further evolution analysis revealed that

474 AtAQPs result from different types of gene duplication, such as $\gamma$ WGD (1), $\beta$ WGD (2),

$475 \alpha$ WGD (8), tandem (2), and transposed (4) (Bowers et al. 2003; Zwiazek et al. 2017b).

476 For poplar, tandem duplication (4) and the recent WGD (20) were the major driving

477 forces for 55 AQP genes (Zou \& Yang 2019).

478 In this study, the 38-member PgrAQP family is comparable to Arabidopsis (33) and

479 Eucalyptus (40), but had a greater number of genes than grape (28). Furthermore, all the

PgrNIP, PgrSIP, and PgrXIP subfamilies were found to have a close relationship with

the corresponding genes in Eucalyptus AQPs, which is consistent with the evolutionary

relationships among the species, suggesting that these AQP subfamilies might have

functional conservation in Myrtales (Qin et al. 2017). It is reasonable to deduce that 
484 WGD events may function as a trigger of PgrAQP family genes expansion. In additional, 485 we identified eight gene duplication events in the PgrAQPs subfamily, including seven 486 segmental duplications and one tandem duplication event (Fig. 2, Table 1).

487 Interestingly, the $\mathrm{Ka} / \mathrm{Ks}$ ratio of the eight duplications was $<1$, indicating that the 488 evolution of the PgrAQP genes is mediated by large-scale purifying selection, similar to 489 the AQP family in B. rapa and wheat (Kayum et al. 2017; Madrid-Espinoza et al. 2018). The synonymous substitution rate was also used to estimate the evolutionary timescale, and the divergence time of duplicated PgrAQP genes occurred 1.77 to 6.97 million years ago, which is in accordance with the divergence time of BrAQPs. This indicates that duplicated divergence of the PgrAQP genes occurred after the triplication events and Myrtales WGD duplication events (Kayum et al. 2017; Qin et al. 2017). that the frequency of gene duplication events was important to the evolution of a species (Flagel \& Wendel 2009; Lynch \& Conery 2000). In cotton and sesame, tandem duplicated genes showed functional differentiation, although they shared structural conservation (Li et al. 2019; WU Xiang-yang 2016). In our study, PgrTIP1.2 and

\subsection{Potential functions of PgrAQP genes}


505

506

507

508

509

510

511

512

513

514

515

516

517

518

519

520

521

522

523

524

525

water absorption of roots and leaves. Furthermore, PIPs can affect photosynthesis by enhancing the diffusion of $\mathrm{CO}_{2}$ in mesophyll tissue of rice and N. tabacum (Flexas et al. 2006; Xu et al. 2019). In this study, PgrPIP subfamily genes had higher expression levels in all analyzed samples compared with other analyzed AQPs. Interestingly, among the RhPIPs, RhPIP1s and RhPIP2s were involved in the expansion of rose petals via an ethylene-dependent pathway (Chen et al. 2013; Ma et al. 2008). The expression analysis showed that PgrPIP1.3 and PgrPIP2.1 had similar expression patterns in pomegranate flowers, suggesting a similar role of PIPs during pomegranate flower development (Fig. 4). In the higher plants, TIPs are widely used as markers for vacuolar compartments and function as transporter for small solutes in various tissue (Bienert et al. 2007; Holm et al. 2005; Liu et al. 2003; Porcel et al. 2018). In Arabidopsis, at least six TIP subfamily numbers, including TIP1;1, TIP1;2, TIP2;1, TIP2;2, TIP2;3, and TIP4;1, showed specific expression patterns in roots (Gattolin et al. 2011). In addition, the rice OsTIP2;1 was only detected in roots (Nguyen et al. 2013). In pomegranate, PgrTIP1.2 and PgrTIP2.3 show higher expression patterns in the roots than other organs, indicated that these gene may specifically participate in the absorption and transport of small solutes, such as $\mathrm{NH}_{4}{ }^{+}$, $\mathrm{H}_{2} \mathrm{O}_{2}$, and urea, in the roots of pomegranate (Bienert et al. 2007; Holm et al. 2005; Liu et al. 2003). In our study, the XIP and majority of NIPs showed lower expression levels than PIPs and TIPs. Interestingly, we found that PgrNIP5.1 was highly expressed in roots. Such specific root expression of NIPs was reported for AtNIP5;1 and HvNIP2;1 (Schnurbusch et al. 2010; Takano et al. 2006), which were involved in boron (B) 
526 homeostasis, indicating that $P g N I P 5 ; 1$ may participate in B absorption and translocation

527 in pomegranate roots.

528 Drought causes tissue dehydration due to an imbalance between plant water

529 uptake and transpiration. Evidence shows that AQPs play an important role in drought

530 tolerance in plants. Ectopically expression of MpPIP2;1 in Arabidopsis has been shown to

531 enhance drought and salinity tolerance. The decrease or increase of the $A Q P S$ transcript

532 levels could prevent water losses or helps plants to direct water flow to specific organ

533 under drought stress. In this study, we found most members of the PgrPIP genes were

534 suppressed by drought stress (Fig 5), including PgrPIP1.1, PgrPIP1.2, PgrPIP1.3,

535 PgrPIP2.1 and PgrPIP2.4, suggested their involvement in reduced water losses in

536 Pomegranate plants. Furthermore, the upregulation of the transcript levels of PgrPIP1.5

537 was observed under 20\% PEG supply condition, indicated that PgrPIP1.5 might play a

538 critical for tolerance to drought in pomegranate.

539 4.4 Identification of candidate $P g r A Q P$ genes involved in pomegranate seed coat

540 development

541 Plant cell expansion is primarily driven by turgor and requires steady water intake,

542 the rate of tissue growth is primarily restricted due to decreasing of turgor (Peret et al.

543 2012; Picaud et al. 2003; Reuscher et al. 2013). For example, in higher plants, the

544 development of a seed coat was primarily initiated by fertilization and driven by cell

545 expansion and growth (Figueiredo et al. 2016). Accordingly, most of the AQPs were

546 reported to be strongly expressed in tissues that can be hydraulically limited during 
547 growth. In Arabidopsis, the specific expression pattern and regulatory mechanism

548 showed that AtTIP1;1 plays a critical role in cell expansion (Beebo et al. 2009; Ludevid et

549 al. 1992). Overexpression of ginseng TIP in Arabidopsis resulted in a significant increase

550 of leaf cell sizes compared with the wild type plants (Lin et al. 2007). In rice, OsPIP1;1 is

551 highly expressed in leaves and roots, and overexpression of OsPIP1;1 exhibited a higher

552 germination rate than the control plants (Liu et al. 2013).

553 In pomegranate, the expanded outer seed coats had higher water accumulation

554 than the rigid inner seed coats in both the hard-seeded cultivar 'Dabenzi' and the soft-

555 seeded cultivar 'Tunisia' (Fig. 6). Accordingly, significant positive correlations were

556 found between the expression level of PgrSIP1.2, PgrPIP1.3, and PgrPIP2.8 and water

557 content in the seed coats (Fig. 7). PgrSIP1.2 was predicted to be localized in the vacuole.

558 Considering that promotion of cell expansion and maintenance of turgor requires the

559 transfer of substantial amounts of water to cells, it is reasonable to proposed that

560 PgrPIP1.3, PgrPIP2.8, and PgrSIP1.2 might be involved in mediating the water

561 accumulation in the inner and outer seed coats of pomegranate.

\section{Conclusions}

563 In this study, a total of $38 \mathrm{AQP}$ genes were identified and their characteristics, including

564 protein physicochemical properties, gene structure, phyletic evolutionary, and

565 expression patterns were studied. These PgrAQP genes are distributed across 9

566 pomegranate chromosomes and divided into five subfamilies. Purifying selection were

567 undergone during the evolution of $\operatorname{Pgr} A Q P$ family genes basing on the syntenic 
568 relationships and duplication events analysis, and a whole-genome duplication event in

569 Myrtales may contribute to the expansion of PgrTIP, PgrSIP, and PgrXIP genes.

570 Furthermore, the high expression of PgrPIP1.3, PgrPIP2.8, and PgrSIP1.2 in seed coats

571 and the positive correlation between transcript levels of PgrAQP genes and the water

572 content revealed these three genes may be the potential candidate genes involving in

573 outer seed coat development. Hence, further studies on functions of this three AQP

574 genes are needed for genetic improvement of outer seed coats in pomegranate.

575

576

577

578

579

580

581

582

583

584

585

586

587

588

589

590

591

592

593

594

595

596

597

598

599

\section{References}

Adams KL, and Wendel JF. 2005. Polyploidy and genome evolution in plants. Curr Opin Plant Biol 8:135-141. 10.1016/j.pbi.2005.01.001

Almasalmeh A, Krenc D, Wu B, and Beitz E. 2014. Structural determinants of the hydrogen peroxide permeability of aquaporins. FEBS J 281:647-656. 10.1111/febs.12653

Artimo P, Jonnalagedda M, Arnold K, Baratin D, Csardi G, de Castro E, Duvaud S, Flegel V, Fortier A, Gasteiger E, Grosdidier A, Hernandez C, loannidis V, Kuznetsov D, Liechti R, Moretti S, Mostaguir K, Redaschi N, Rossier G, Xenarios I, and Stockinger H. 2012. ExPASy: SIB bioinformatics resource portal. Nucleic Acids Res 40:W597-603. 10.1093/nar/gks400

Ayadi M, Cavez D, Miled N, Chaumont F, Masmoudi K. Identification and characterization of two plasma membran e aquaporins in durum wheat (Triticum turgidum L. subsp. durum) and their role in abiotic stress toleranc e. Plant Physiol Biochem. 2011 Sep;49(9):102939. doi: 10.1016/j.plaphy.2011.06.002. Epub 2011 Jun 17. PMID: 21723739.

Azad AK, Ahmed J, Alum MA, Hasan MM, Ishikawa T, Sawa Y, and Katsuhara M. 2016. Genome-Wide Characterization of Major Intrinsic Proteins in Four Grass Plants and Their Non-Aqua Transport Selectivity Profiles with Comparative Perspective. PLoS One 11:e0157735. 10.1371/journal.pone.0157735

Azad AK, Sawa Y, Ishikawa T, and Shibata H. 2004. Phosphorylation of plasma membrane aquaporin regulates temperature-dependent opening of tulip petals. Plant Cell Physiol 45:608-617. 10.1093/pcp/pch069

Bailey TL, Boden M, Buske FA, Frith M, Grant CE, Clementi L, Ren J, Li WW, and Noble WS. 2009. MEME SUITE: tools for motif discovery and searching. Nucleic Acids Res 37:W202-208. 10.1093/nar/gkp335

Beebo A, Thomas D, Der C, Sanchez L, Leborgne-Castel N, Marty F, Schoefs B, and Bouhidel K. 2009. Life with and without AtTIP1;1, an Arabidopsis aquaporin preferentially localized in the apposing tonoplasts of adjacent vacuoles. Plant Mol Biol 70:193-209. 10.1007/s11103-009-9465-2

Bienert GP, Moller AL, Kristiansen KA, Schulz A, Moller IM, Schjoerring JK, and Jahn TP. 2007. Specific aquaporins 
600

601

602

603

604

605

606

607

608

609

610

611

612

613

614

615

616

617

618

619

620

621

622

623

624

625

626

627

628

629

630

631

632

633

634

635

636

637

638

639

640

facilitate the diffusion of hydrogen peroxide across membranes. J Biol Chem 282:1183-1192. 10.1074/jbc.M603761200

Bowers JE, Chapman BA, Rong J, and Paterson AH. 2003. Unravelling angiosperm genome evolution by phylogenetic analysis of chromosomal duplication events. Nature 422:433-438. 10.1038/nature01521

Chaumont F, Barrieu F, Wojcik E, Chrispeels MJ, and Jung R. 2001. Aquaporins constitute a large and highly divergent protein family in maize. Plant Physiol 125:1206-1215. 10.1104/pp.125.3.1206

Chen C, Chen H, Zhang Y, Thomas HR, Frank MH, He Y, and Xia R. 2020. TBtools: An Integrative Toolkit Developed for Interactive Analyses of Big Biological Data. Mol Plant 13:1194-1202. 10.1016/j.molp.2020.06.009

Chen W, Yin X, Wang L, Tian J, Yang R, Liu D, Yu Z, Ma N, and Gao J. 2013. Involvement of rose aquaporin RhPIP1;1 in ethylene-regulated petal expansion through interaction with RhPIP2;1. Plant Mol Biol 83:219-233. 10.1007/s11103-013-0084-6

Danielson JA, and Johanson U. 2008. Unexpected complexity of the aquaporin gene family in the moss Physcomitrella patens. BMC Plant Biol 8:45. 10.1186/1471-2229-8-45

Danielson JA, and Johanson U. 2010. Phylogeny of major intrinsic proteins. Adv Exp Med Biol 679:19-31. 10.1007/978-1-4419-6315-4_2

De Rosa A, Watson-Lazowski A, Evans JR, and Groszmann M. 2020. Genome-wide identification and characterisation of Aquaporins in Nicotiana tabacum and their relationships with other Solanaceae species. BMC Plant Biol 20:266. 10.1186/s12870-020-02412-5

Deokar AA, and Tar'an B. 2016. Genome-Wide Analysis of the Aquaporin Gene Family in Chickpea (Cicer arietinum L.). Front Plant Sci 7:1802. 10.3389/fpls.2016.01802

Deshmukh RK, Vivancos J, Guérin V, Sonah H, Labbé C, Belzile F, and Bélanger RR. 2013. Identification and functional characterization of silicon transporters in soybean using comparative genomics of major intrinsic proteins in Arabidopsis and rice. Plant Mol Biol 83:303-315. 10.1007/s11103-013-0087-3

Deshmukh RK, Vivancos J, Ramakrishnan G, Guérin V, Carpentier G, Sonah H, Labbé C, Isenring P, Belzile FJ, and Bélanger RR. 2015. A precise spacing between the NPA domains of aquaporins is essential for silicon permeability in plants. Plant J 83:489-500. 10.1111/tpj.12904

Edlund AF, Swanson R, and Preuss D. 2004. Pollen and stigma structure and function: the role of diversity in pollination. Plant Cell 16 Suppl:S84-97. 10.1105/tpc.015800

Eisenbarth DA, and Weig AR. 2005. Dynamics of aquaporins and water relations during hypocotyl elongation in Ricinus communis L. seedlings. J Exp Bot 56:1831-1842. 10.1093/jxb/eri173

Faize M, Fumanal B, Luque F, Ramirez-Tejero JA, Zou Z, Qiao X, Faize L, Gousset-Dupont A, Roeckel-Drevet P, Label $P$, and Venisse JS. 2020. Genome Wild Analysis and Molecular Understanding of the Aquaporin Diversity in Olive Trees (Olea Europaea L.). Int J Mol Sci 21. 10.3390/ijms21114183

Figueiredo DD, Batista RA, Roszak PJ, Hennig L, and Kohler C. 2016. Auxin production in the endosperm drives seed coat development in Arabidopsis. Elife 5. 10.7554/eLife.20542

Finn RD, Bateman A, Clements J, Coggill P, Eberhardt RY, Eddy SR, Heger A, Hetherington K, Holm L, Mistry J, Sonnhammer EL, Tate J, and Punta M. 2014. Pfam: the protein families database. Nucleic Acids Res 42:D222-230. 10.1093/nar/gkt1223

Flagel LE, and Wendel JF. 2009. Gene duplication and evolutionary novelty in plants. New Phytol 183:557-564. 10.1111/j.1469-8137.2009.02923.x

Flexas J, Ribas-Carbo M, Hanson DT, Bota J, Otto B, Cifre J, McDowell N, Medrano H, and Kaldenhoff R. 2006.

Peer) reviewing PDF | (2020:12:56609:2:0:NEW 8 Jun 2021) 
641

642

643

644

645

646

647

648

649

650

651

652

653

654

655

656

657

658

659

660

661

662

663

664

665

666

667

668

669

670

671

672

673

674

675

676

677

678

679

680

681

Tobacco aquaporin NtAQP1 is involved in mesophyll conductance to CO2 in vivo. Plant J 48:427-439. 10.1111/j.1365-313X.2006.02879.x

Fouquet R, Léon C, Ollat N, and Barrieu F. 2008. Identification of grapevine aquaporins and expression analysis in developing berries. Plant Cell Reports 27:1541-1550. 10.1007/s00299-008-0566-1

Froger A, Tallur B, Thomas D, and Delamarche C. 1998. Prediction of functional residues in water channels and related proteins. Protein Sci 7:1458-1468. 10.1002/pro.5560070623

Gattolin S, Sorieul M, and Frigerio L. 2011. Mapping of tonoplast intrinsic proteins in maturing and germinating Arabidopsis seeds reveals dual localization of embryonic TIPs to the tonoplast and plasma membrane. Mol Plant 4:180-189. 10.1093/mp/ssq051

Gu Z, Cavalcanti A, Chen FC, Bouman P, and Li WH. 2002. Extent of gene duplication in the genomes of Drosophila, nematode, and yeast. Mol Biol Evol 19:256-262. 10.1093/oxfordjournals.molbev.a004079

Gupta AB, and Sankararamakrishnan R. 2009. Genome-wide analysis of major intrinsic proteins in the tree plant Populus trichocarpa: characterization of XIP subfamily of aquaporins from evolutionary perspective. BMC Plant Biol 9:134. 10.1186/1471-2229-9-134

Holm LM, Jahn TP, Moller AL, Schjoerring JK, Ferri D, Klaerke DA, and Zeuthen T. 2005. NH3 and NH4+ permeability in aquaporin-expressing Xenopus oocytes. Pflugers Arch 450:415-428. 10.1007/s00424-005-1399-1

Hove RM, and Bhave M. 2011. Plant aquaporins with non-aqua functions: deciphering the signature sequences. Plant Mol Biol 75:413-430. 10.1007/s11103-011-9737-5

Hu B, Jin J, Guo AY, Zhang H, Luo J, and Gao G. 2015a. GSDS 2.0: an upgraded gene feature visualization server. Bioinformatics 31:1296-1297. 10.1093/bioinformatics/btu817

Hu W, Hou X, Huang C, Yan Y, Tie W, Ding Z, Wei Y, Liu J, Miao H, Lu Z, Li M, Xu B, and Jin Z. 2015b. Genome-Wide Identification and Expression Analyses of Aquaporin Gene Family during Development and Abiotic Stress in Banana. Int J Mol Sci 16:19728-19751. 10.3390/ijms160819728

Hu W, Hua X, Zhang Q, Wang J, Shen Q, Zhang X, Wang K, Yu Q, Lin YR, Ming R, and Zhang J. 2018. New insights into the evolution and functional divergence of the SWEET family in Saccharum based on comparative genomics. BMC Plant Biol 18:270. 10.1186/s12870-018-1495-y

Ivanov, II, Loktyushkin AV, Gus'kova RA, Vasil'ev NS, Fedorov GE, and Rubin AB. 2007. Oxygen channels of erythrocyte membrane. Dokl Biochem Biophys 414:137-140. 10.1134/s160767290703012x

Jiao Y, Leebens-Mack J, Ayyampalayam S, Bowers JE, McKain MR, McNeal J, Rolf M, Ruzicka DR, Wafula E, Wickett NJ, Wu X, Zhang Y, Wang J, Zhang Y, Carpenter EJ, Deyholos MK, Kutchan TM, Chanderbali AS, Soltis PS, Stevenson DW, McCombie R, Pires JC, Wong GK, Soltis DE, and Depamphilis CW. 2012. A genome triplication associated with early diversification of the core eudicots. Genome Biol 13:R3. 10.1186/gb2012-13-1-r3

Jiao Y, Li J, Tang H, and Paterson AH. 2014. Integrated syntenic and phylogenomic analyses reveal an ancient genome duplication in monocots. Plant Cell 26:2792-2802. 10.1105/tpc.114.127597

Johanningsmeier SD, and Harris GK. 2011. Pomegranate as a Functional Food and Nutraceutical Source. Annual Review of Food Science and Technology 2:181-201. 10.1146/annurev-food-030810-153709

Kaldenhoff R, and Fischer M. 2006. Functional aquaporin diversity in plants. Biochim Biophys Acta 1758:1134-1141. 10.1016/j.bbamem.2006.03.012

Kayum MA, Park JI, Nath UK, Biswas MK, Kim HT, and Nou IS. 2017. Genome-wide expression profiling of aquaporin genes confer responses to abiotic and biotic stresses in Brassica rapa. BMC Plant Biol 17:23.

PeerJ reviewing PDF | (2020:12:56609:2:0:NEW 8 Jun 2021) 
682

683

684

685

686

687

688

689

690

691

692

693

694

695

696

697

698

699

700

701

702

703

704

705

706

707

708

709

710

711

712

713

714

715

716

717

718

719

720

721

722

10.1186/s12870-017-0979-5

Khabudaev KV, Petrova DP, Grachev MA, and Likhoshway YV. 2014. A new subfamily LIP of the major intrinsic proteins. BMC Genomics 15:173. 10.1186/1471-2164-15-173

Kumar S, Stecher G, and Tamura K. 2016. MEGA7: Molecular Evolutionary Genetics Analysis Version 7.0 for Bigger Datasets. Mol Biol Evol 33:1870-1874. 10.1093/molbev/msw054

Lee JK, Kozono D, Remis J, Kitagawa Y, Agre P, and Stroud RM. 2005. Structural basis for conductance by the archaeal aquaporin AqpM at 1.68 A. Proc Natl Acad Sci U S A 102:18932-18937. 10.1073/pnas.0509469102

Letunic I, Khedkar S, and Bork P. 2020. SMART: recent updates, new developments and status in 2020. Nucleic Acids Res. 10.1093/nar/gkaa937

Li W, Zhang D, Zhu G, Mi X, and Guo W. 2019. Combining genome-wide and transcriptome-wide analyses reveal the evolutionary conservation and functional diversity of aquaporins in cotton. BMC Genomics 20:538. 10.1186/s12864-019-5928-2

Lian HL, Yu X, Ye Q, Ding X, Kitagawa Y, Kwak SS, Su WA, and Tang ZC. 2004. The role of aquaporin RWC3 in drought avoidance in rice. Plant Cell Physiol 45:481-489. 10.1093/pcp/pch058

Lin W, Peng Y, Li G, Arora R, Tang Z, Su W, and Cai W. 2007. Isolation and functional characterization of PgTIP1, a hormone-autotrophic cells-specific tonoplast aquaporin in ginseng. J Exp Bot 58:947-956. 10.1093/jxb/erl255

Liu C, Fukumoto T, Matsumoto T, Gena P, Frascaria D, Kaneko T, Katsuhara M, Zhong S, Sun X, Zhu Y, Iwasaki I, Ding X, Calamita G, and Kitagawa Y. 2013. Aquaporin OsPIP1;1 promotes rice salt resistance and seed germination. Plant Physiol Biochem 63:151-158. 10.1016/j.plaphy.2012.11.018

Liu HY, Yu X, Cui DY, Sun MH, Sun WN, Tang ZC, Kwak SS, and Su WA. 2007. The role of water channel proteins and nitric oxide signaling in rice seed germination. Cell Res 17:638-649. 10.1038/cr.2007.34

Liu LH, Ludewig U, Gassert B, Frommer WB, and von Wiren N. 2003. Urea transport by nitrogen-regulated tonoplast intrinsic proteins in Arabidopsis. Plant Physiol 133:1220-1228. 10.1104/pp.103.027409

Ludevid D, Hofte H, Himelblau E, and Chrispeels MJ. 1992. The Expression Pattern of the Tonoplast Intrinsic Protein gamma-TIP in Arabidopsis thaliana Is Correlated with Cell Enlargement. Plant Physiol 100:1633-1639. 10.1104/pp.100.4.1633

Luo X, Li H, Wu Z, Yao W, Zhao P, Cao D, Yu H, Li K, Poudel K, Zhao D, Zhang F, Xia X, Chen L, Wang Q, Jing D, and Cao S. 2020. The pomegranate (Punica granatum L.) draft genome dissects genetic divergence between soft- and hard-seeded cultivars. Plant Biotechnol J 18:955-968. 10.1111/pbi.13260

Lynch M, and Conery JS. 2000. The evolutionary fate and consequences of duplicate genes. Science 290:1151-1155. 10.1126/science.290.5494.1151

Ma N, Xue J, Li Y, Liu X, Dai F, Jia W, Luo Y, and Gao J. 2008. Rh-PIP2;1, a rose aquaporin gene, is involved in ethylene-regulated petal expansion. Plant Physiol 148:894-907. 10.1104/pp.108.120154

Madrid-Espinoza J, Brunel-Saldias N, Guerra FP, Gutierrez A, and Del Pozo A. 2018. Genome-Wide Identification and Transcriptional Regulation of Aquaporin Genes in Bread Wheat (Triticum aestivum L.) under Water Stress. Genes (Basel) 9. 10.3390/genes9100497

Martins Cde P, Pedrosa AM, Du D, Goncalves LP, Yu Q, Gmitter FG, Jr., and Costa MG. 2015. Genome-Wide Characterization and Expression Analysis of Major Intrinsic Proteins during Abiotic and Biotic Stresses in Sweet Orange (Citrus sinensis L. Osb.). PLoS One 10:e0138786. 10.1371/journal.pone.0138786

PeerJ reviewing PDF | (2020:12:56609:2:0:NEW 8 Jun 2021) 
723

724

725

726

727

728

729

730

731

732

733

734

735

736

737

738

739

740

741

742

743

744

745

746

747

748

749

750

751

752

753

754

755

756

757

758

759

760

761

762

763

Myburg AA, Grattapaglia D, Tuskan GA, Hellsten U, Hayes RD, Grimwood J, Jenkins J, Lindquist E, Tice H, Bauer D, Goodstein DM, Dubchak I, Poliakov A, Mizrachi E, Kullan AR, Hussey SG, Pinard D, van der Merwe K, Singh P, van Jaarsveld I, Silva-Junior OB, Togawa RC, Pappas MR, Faria DA, Sansaloni CP, Petroli CD, Yang X, Ranjan P, Tschaplinski TJ, Ye CY, Li T, Sterck L, Vanneste K, Murat F, Soler M, Clemente HS, Saidi N, CassanWang H, Dunand C, Hefer CA, Bornberg-Bauer E, Kersting AR, Vining K, Amarasinghe V, Ranik M, Naithani S, Elser J, Boyd AE, Liston A, Spatafora JW, Dharmwardhana P, Raja R, Sullivan C, Romanel E, Alves-Ferreira M, Kulheim C, Foley W, Carocha V, Paiva J, Kudrna D, Brommonschenkel SH, Pasquali G, Byrne M, Rigault P, Tibbits J, Spokevicius A, Jones RC, Steane DA, Vaillancourt RE, Potts BM, Joubert F, Barry K, Pappas GJ, Strauss SH, Jaiswal P, Grima-Pettenati J, Salse J, Van de Peer Y, Rokhsar DS, and Schmutz J. 2014. The genome of Eucalyptus grandis. Nature 510:356-362. 10.1038/nature13308

Nguyen MX, Moon S, and Jung KH. 2013. Genome-wide expression analysis of rice aquaporin genes and development of a functional gene network mediated by aquaporin expression in roots. Planta 238:669681. 10.1007/s00425-013-1918-9

Niu J, Cao D, Li H, Xue H, Chen L, Liu B, and Cao S. 2018. Quantitative proteomics of pomegranate varieties with contrasting seed hardness during seed development stages. Tree Genetics \& Genomes 14:14. 10.1007/s11295-018-1229-1

Patel C, Dadhaniya P, Hingorani L, and Soni MG. 2008. Safety assessment of pomegranate fruit extract: acute and subchronic toxicity studies. Food Chem Toxicol 46:2728-2735. 10.1016/j.fct.2008.04.035

Peret B, Li G, Zhao J, Band LR, Voss U, Postaire O, Luu DT, Da Ines O, Casimiro I, Lucas M, Wells DM, Lazzerini L, Nacry P, King JR, Jensen OE, Schaffner AR, Maurel C, and Bennett MJ. 2012. Auxin regulates aquaporin function to facilitate lateral root emergence. Nat Cell Biol 14:991-998. 10.1038/ncb2573

Picaud S, Becq F, Dedaldechamp F, Ageorges A, and Delrot S. 2003. Cloning and expression of two plasma membrane aquaporins expressed during the ripening of grape berry. Funct Plant Biol 30:621-630. 10.1071/FP02116

Pommerrenig B, Diehn TA, and Bienert GP. 2015. Metalloido-porins: Essentiality of Nodulin 26-like intrinsic proteins in metalloid transport. Plant Sci 238:212-227. 10.1016/j.plantsci.2015.06.002

Porcel R, Bustamante A, Ros R, Serrano R, and Mulet Salort JM. 2018. BvCOLD1: A novel aquaporin from sugar beet (Beta vulgaris L.) involved in boron homeostasis and abiotic stress. Plant Cell Environ 41:2844-2857. 10.1111/pce.13416

Qiao X, Li M, Li L, Yin H, Wu J, and Zhang S. 2015. Genome-wide identification and comparative analysis of the heat shock transcription factor family in Chinese white pear (Pyrus bretschneideri) and five other Rosaceae species. BMC Plant Biol 15:12. 10.1186/s12870-014-0401-5

Qin G, Liu C, Li J, Qi Y, Gao Z, Zhang X, Yi X, Pan H, Ming R, and Xu Y. 2020. Diversity of metabolite accumulation patterns in inner and outer seed coats of pomegranate: exploring their relationship with genetic mechanisms of seed coat development. Hortic Res 7:10. 10.1038/s41438-019-0233-4

Qin G, Xu C, Ming R, Tang H, Guyot R, Kramer EM, Hu Y, Yi X, Qi Y, Xu X, Gao Z, Pan H, Jian J, Tian Y, Yue Z, and Xu Y. 2017. The pomegranate (Punica granatum L.) genome and the genomics of punicalagin biosynthesis. Plant J 91:1108-1128. 10.1111/tpj.13625

Quigley F, Rosenberg JM, Shachar-Hill Y, and Bohnert HJ. 2002. From genome to function: the Arabidopsis aquaporins. Genome Biol 3:RESEARCH0001. 10.1186/gb-2001-3-1-research0001

Reuscher S, Akiyama M, Mori C, Aoki K, Shibata D, and Shiratake K. 2013. Genome-wide identification and

Peer) reviewing PDF | (2020:12:56609:2:0:NEW 8 Jun 2021) 
expression analysis of aquaporins in tomato. PLoS One 8:e79052. 10.1371/journal.pone.0079052

Sakurai J, Ishikawa F, Yamaguchi T, Uemura M, and Maeshima M. 2005. Identification of 33 rice aquaporin genes and analysis of their expression and function. Plant Cell Physiol 46:1568-1577. 10.1093/pcp/pci172

Schnurbusch T, Hayes J, Hrmova M, Baumann U, Ramesh SA, Tyerman SD, Langridge P, and Sutton T. 2010. Boron Toxicity Tolerance in Barley through Reduced Expression of the Multifunctional Aquaporin HvNIP2;1. 153:1706-1715. 10.1104/pp.110.158832 \%J Plant Physiology

Schuurmans JA, van Dongen JT, Rutjens BP, Boonman A, Pieterse CM, Borstlap AC. Members of the aquaporin famil $\mathrm{y}$ in the developing pea seed coat include representatives of the PIP, TIP, and NIP subfamilies. Plant Mol Bi ol. 2003 Nov;53(5):633-45. doi: 10.1023/B:PLAN.0000019070.60954.77. PMID: 15010602.

Shivaraj SM, Deshmukh R, Sonah H, and Belanger RR. 2019. Identification and characterization of aquaporin genes in Arachis duranensis and Arachis ipaensis genomes, the diploid progenitors of peanut. BMC Genomics 20:222. 10.1186/s12864-019-5606-4

Soto G, Alleva K, Mazzella MA, Amodeo G, and Muschietti JP. 2008. AtTIP1;3 and AtTIP5;1, the only highly expressed Arabidopsis pollen-specific aquaporins, transport water and urea. FEBS Lett 582:4077-4082. 10.1016/j.febslet.2008.11.002

Soto G, Fox R, Ayub N, Alleva K, Guaimas F, Erijman EJ, Mazzella A, Amodeo G, and Muschietti J. 2010. TIP5;1 is an aquaporin specifically targeted to pollen mitochondria and is probably involved in nitrogen remobilization in Arabidopsis thaliana. Plant J 64:1038-1047. 10.1111/j.1365-313X.2010.04395.x

Suga N, Takada H, Nomura A, Ohga S, Ishii E, Ihara K, Ohshima K, and Hara T. 2002. Perforin defects of primary haemophagocytic lymphohistiocytosis in Japan. Br J Haematol 116:346-349. 10.1046/j.13652141.2002.03266.x

Takano J, Wada M, Ludewig U, Schaaf G, von Wiren N, and Fujiwara T. 2006. The Arabidopsis major intrinsic protein NIP5;1 is essential for efficient boron uptake and plant development under boron limitation. Plant Cell 18:1498-1509. 10.1105/tpc.106.041640

Terashima I, and Ono K. 2002. Effects of $\mathrm{HgCl}(2)$ on $\mathrm{CO}(2)$ dependence of leaf photosynthesis: evidence indicating involvement of aquaporins in $\mathrm{CO}(2)$ diffusion across the plasma membrane. Plant Cell Physiol 43:70-78. 10.1093/pcp/pcf001

Tornroth-Horsefield S, Wang Y, Hedfalk K, Johanson U, Karlsson M, Tajkhorshid E, Neutze R, and Kjellbom P. 2006. Structural mechanism of plant aquaporin gating. Nature 439:688-694. 10.1038/nature04316

Uçar S, and Karagöz S. 2009. The slow pyrolysis of pomegranate seeds: The effect of temperature on the product yields and bio-oil properties. Journal of Analytical and Applied Pyrolysis 84:151-156. https://doi.org/10.1016/j.jaap.2009.01.005

Vander Willigen C, Postaire O, Tournaire-Roux C, Boursiac Y, and Maurel C. 2006. Expression and inhibition of aquaporins in germinating Arabidopsis seeds. Plant Cell Physiol 47:1241-1250. 10.1093/pcp/pcj094

Wallace IS, and Roberts DM. 2004. Homology modeling of representative subfamilies of Arabidopsis major intrinsic proteins. Classification based on the aromatic/arginine selectivity filter. Plant Physiol 135:1059-1068. 10.1104/pp.103.033415

Wang L, Guo K, Li Y, Tu Y, Hu H, Wang B, Cui X, and Peng L. 2010. Expression profiling and integrative analysis of the CESA/CSL superfamily in rice. BMC Plant Biol 10:282. 10.1186/1471-2229-10-282

WU Xiang-yang CC-z, Lü Gao-qiang, WANG Xin-yu. 2016. Identification and Characterization of the AQP Gene Family in Sesame. 49:1844-1858. 10.3864/j.issn.0578-1752.2016.10.002 
805

806

807

808

809

810

811

812

813

814

815

816

817

818

819

820

821

822

823

824

825

826

827

828

829

830

831

832

833

834

835

836

837

Wudick MM, Luu DT, Tournaire-Roux C, Sakamoto W, and Maurel C. 2014. Vegetative and sperm cell-specific aquaporins of Arabidopsis highlight the vacuolar equipment of pollen and contribute to plant reproduction. Plant Physiol 164:1697-1706. 10.1104/pp.113.228700

Xu F, Wang K, Yuan W, Xu W, Liu S, Kronzucker HJ, Chen G, Miao R, Zhang M, Ding M, Xiao L, Kai L, Zhang J, and Zhu Y. 2019. Overexpression of rice aquaporin OsPIP1;2 improves yield by enhancing mesophyll CO2 conductance and phloem sucrose transport. Journal of Experimental Botany 70:671-681. 10.1093/jxb/ery386

Yang S, Zhang X, Yue JX, Tian D, and Chen JQ. 2008. Recent duplications dominate NBS-encoding gene expansion in two woody species. Mol Genet Genomics 280:187-198. 10.1007/s00438-008-0355-0

Yasui M, Hazama A, Kwon TH, Nielsen S, Guggino WB, and Agre P. 1999. Rapid gating and anion permeability of an intracellular aquaporin. Nature 402:184-187. 10.1038/46045

Zarei A, Zamani Z, Fatahi R, Mousavi A, Salami SA, Avila C, and Cánovas FM. 2016. Differential expression of cell wall related genes in the seeds of soft- and hard-seeded pomegranate genotypes. Scientia Horticulturae 205:7-16. https://doi.org/10.1016/j.scienta.2016.03.043

Zhang DY, Ali Z, Wang CB, Xu L, Yi JX, Xu ZL, Liu XQ, He XL, Huang YH, Khan IA, Trethowan RM, and Ma HX. 2013. Genome-wide sequence characterization and expression analysis of major intrinsic proteins in soybean (Glycine max L.). PLoS One 8:e56312. 10.1371/journal.pone.0056312

Zhou Y, Setz N, Niemietz C, Qu H, Offler CE, Tyerman SD, and Patrick JW. 2007a. Aquaporins and unloading of phloem-imported water in coats of developing bean seeds. Plant Cell Environ 30:1566-1577. 10.1111/j.1365-3040.2007.01732.x

ZHOU Y, SETZ N, NIEMIETZ C, QU H, OFFLER CE, TYERMAN SD, and PATRICK JW. 2007b. Aquaporins and unloading of phloem-imported water in coats of developing bean seeds. 30:1566-1577. https://doi.org/10.1111/j.1365-3040.2007.01732.x

Zhou Y, Tao J, Ahammed GJ, Li J, and Yang Y. 2019. Genome-wide identification and expression analysis of aquaporin gene family related to abiotic stress in watermelon. Genome 62:643-656. 10.1139/gen-20190061

Zou Z, and Yang J. 2019. Genome-wide comparison reveals divergence of cassava and rubber aquaporin family genes after the recent whole-genome duplication. BMC Genomics 20:380. 10.1186/s12864-019-5780-4

Zwiazek J, Xu H, Tan X, Navarro-Ródenas A, and Morte A. 2017a. Significance of oxygen transport through aquaporins. Scientific Reports 7:40411. 10.1038/srep40411

Zwiazek JJ, Xu H, Tan X, Navarro-Rodenas A, and Morte A. 2017b. Significance of oxygen transport through aquaporins. Sci Rep 7:40411. 10.1038/srep40411

Peer] reviewing PDF | (2020:12:56609:2:0:NEW 8 Jun 2021) 


\section{Figure 1}

Phylogenetic analysis of AQP proteins from pomegranate, grape, Arabidopsis, Populus trichocarpa and Eucalyptus.

The tree was generated by the neighbor-joining (NJ) method in MEGA 7.0 with 1,000 bootstrap replicates. Different background colors indicate the different subfamilies of the AQP proteins.

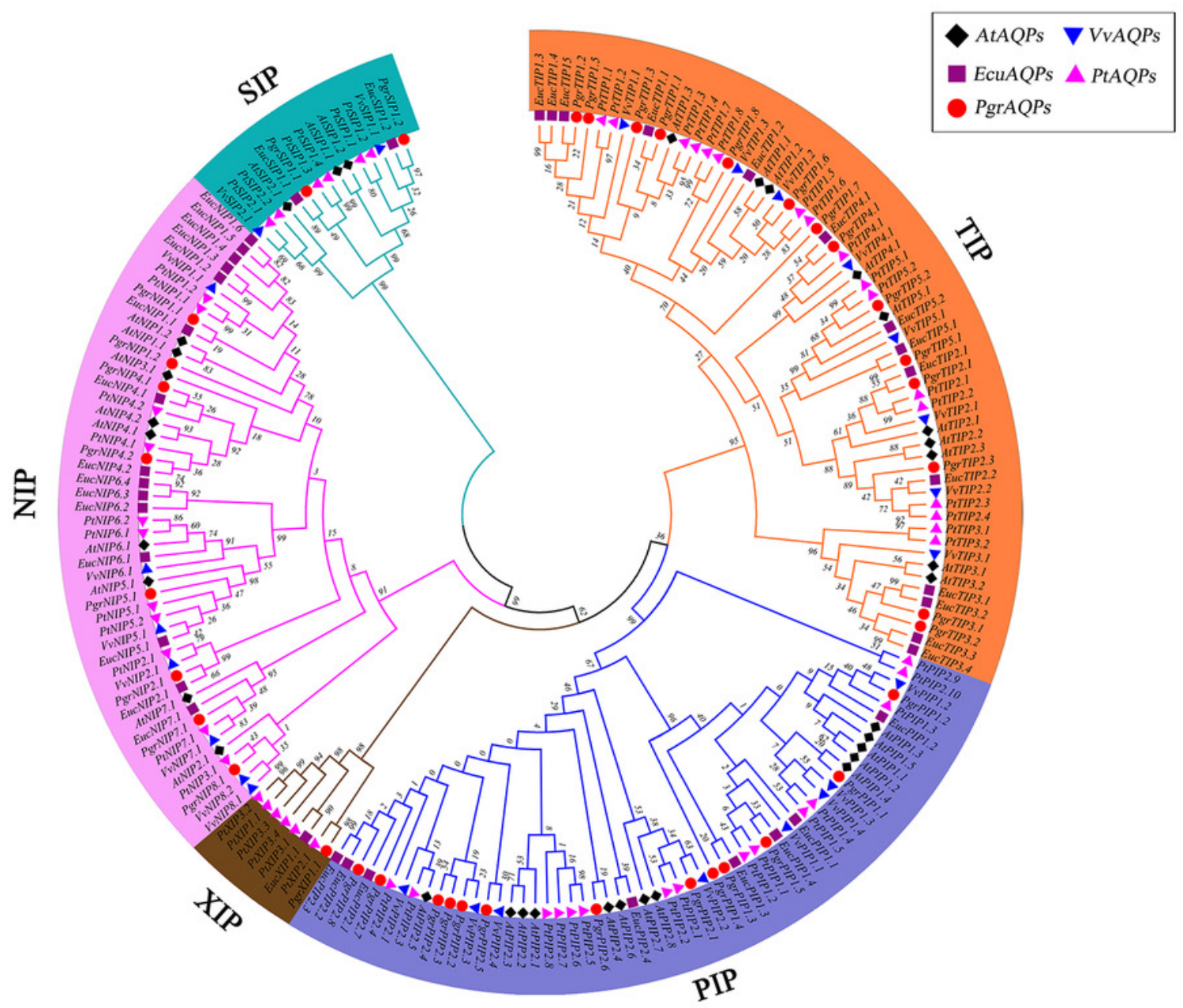




\section{Figure 2}

Analysis of chromosomal locations and syntenic relationships of PgrAQP genes.

The AQP genes in pomegranate were mapped to different chromosomes using TBtools, and AQP genes in red and blue represent genes with segmental and tandem duplications, respectively.

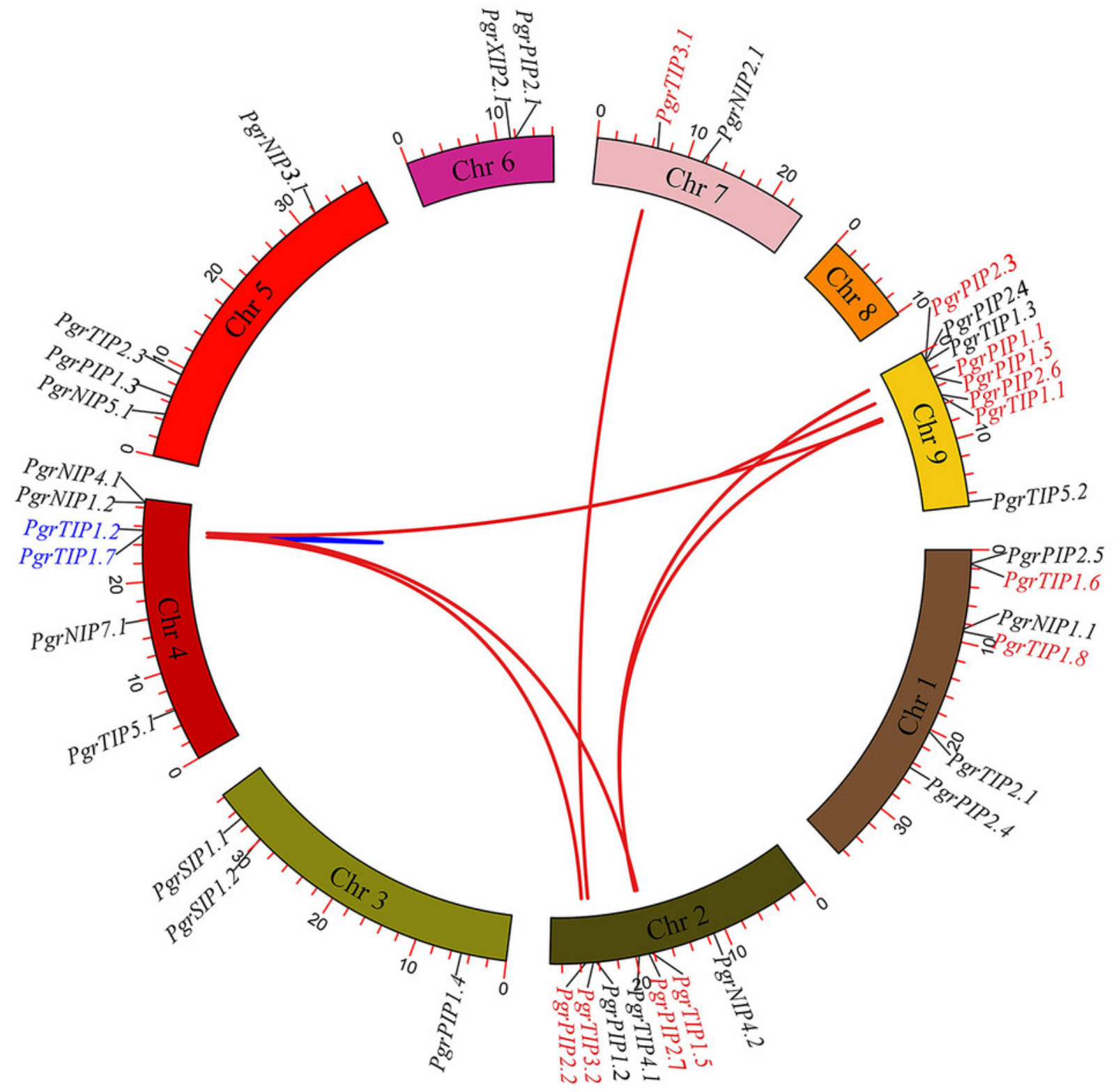


Figure 3

Synteny analysis of PgrAQP genes between pomegranate and three plant species.

(A) Pomegranate and A. thaliana, (B) pomegranate and Vitis vinifera, (C) pomegranate and Eucalyptus grandis. The gray lines indicated collinearity between pomegranate and other species. The red lines highlight the syntenic AQP gene pairs. The chromosome name is indicated at the top of every chromosome.

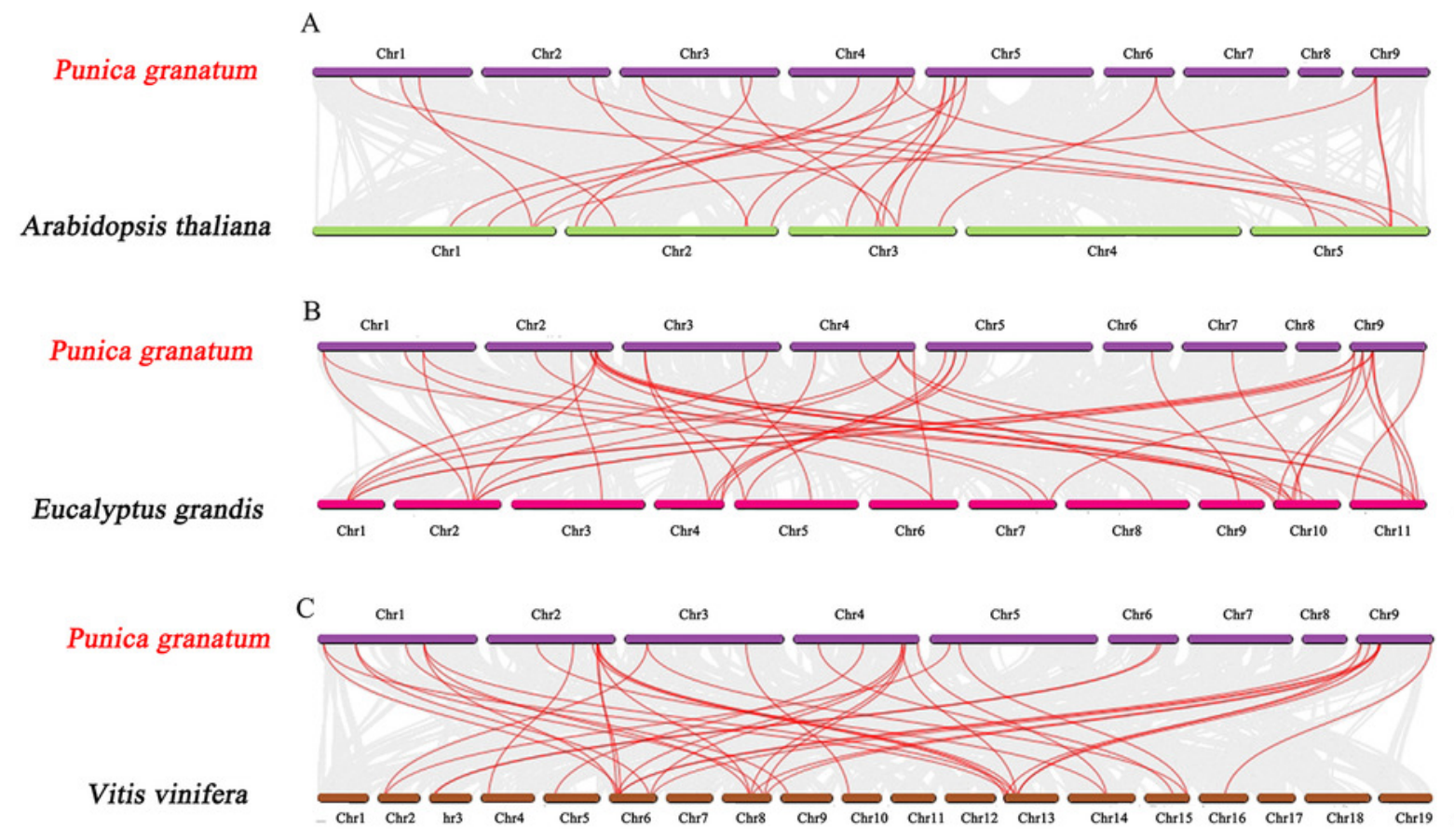




\section{Figure 4}

Expression analysis of the PgrAQP genes in pomegranate.

(A) Expression profile of PgrAQP genes in the cultivated pomegranate cultivar 'Dabenzi,' including roots, flowers, leaves, and three stages of the peel, inner, and outer seed coats (50, 95, and 140 days after pollination). (B) Expression profiling of PgrAQP genes at different developmental stages of the seed coats in pomegranate. The abbreviations are as follows: D: P. granatum 'Dabenzi,' T: P. granatum 'Tunisia,' O: Outer seed coat, I: Inner seed coat. The number represents the number of days after pollination (DAP). The heat map was generated using TBtools. Expression data were calculated with log2 normalization based on FPKM values.

(A)

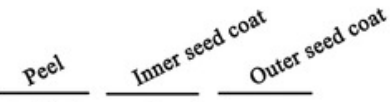

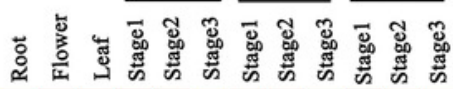

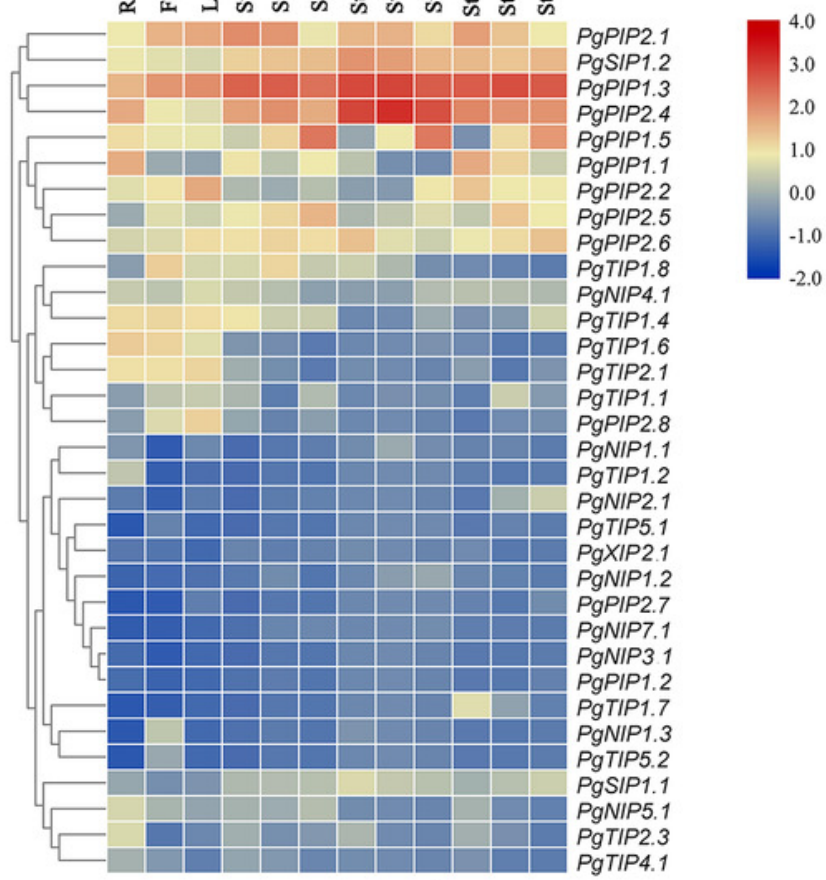

(B)

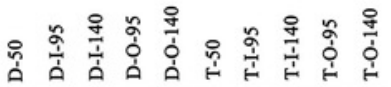

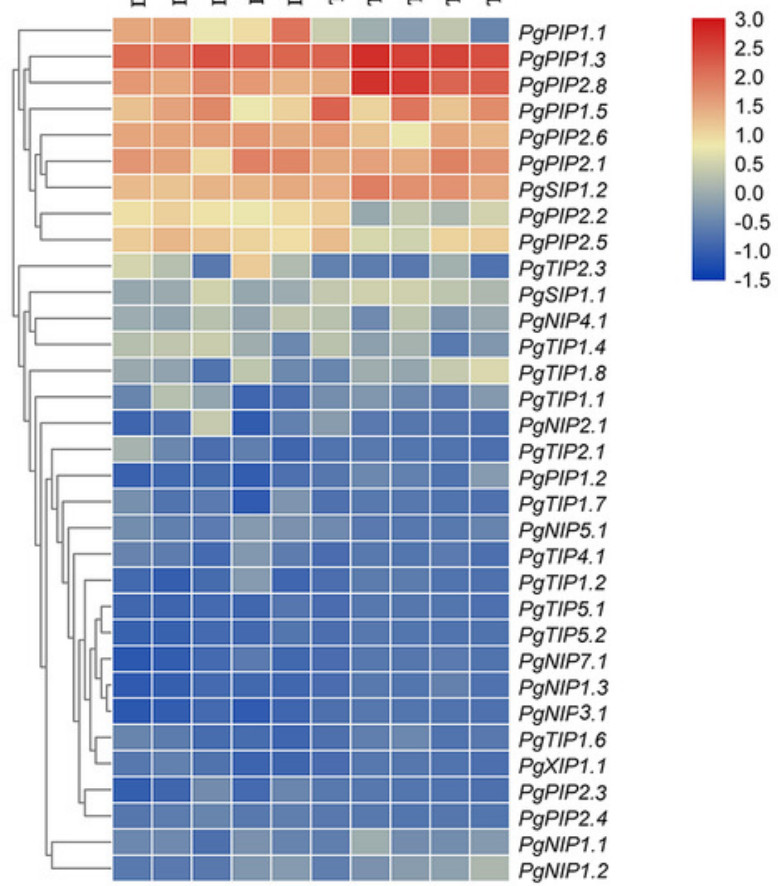


Figure 5

Expression profiles of six Pomegranate PIPs roots in response to water deficit qRT-PCR was performed to determined the relative transcript level for the six PgrPIP genes. Relative expression level was normalized relative to untreated control group (0 h PEG treatment). Error bar represent SE of three independent biological replicated. Asterisk indicate significant differences, $* \mathrm{P}<0.05$.

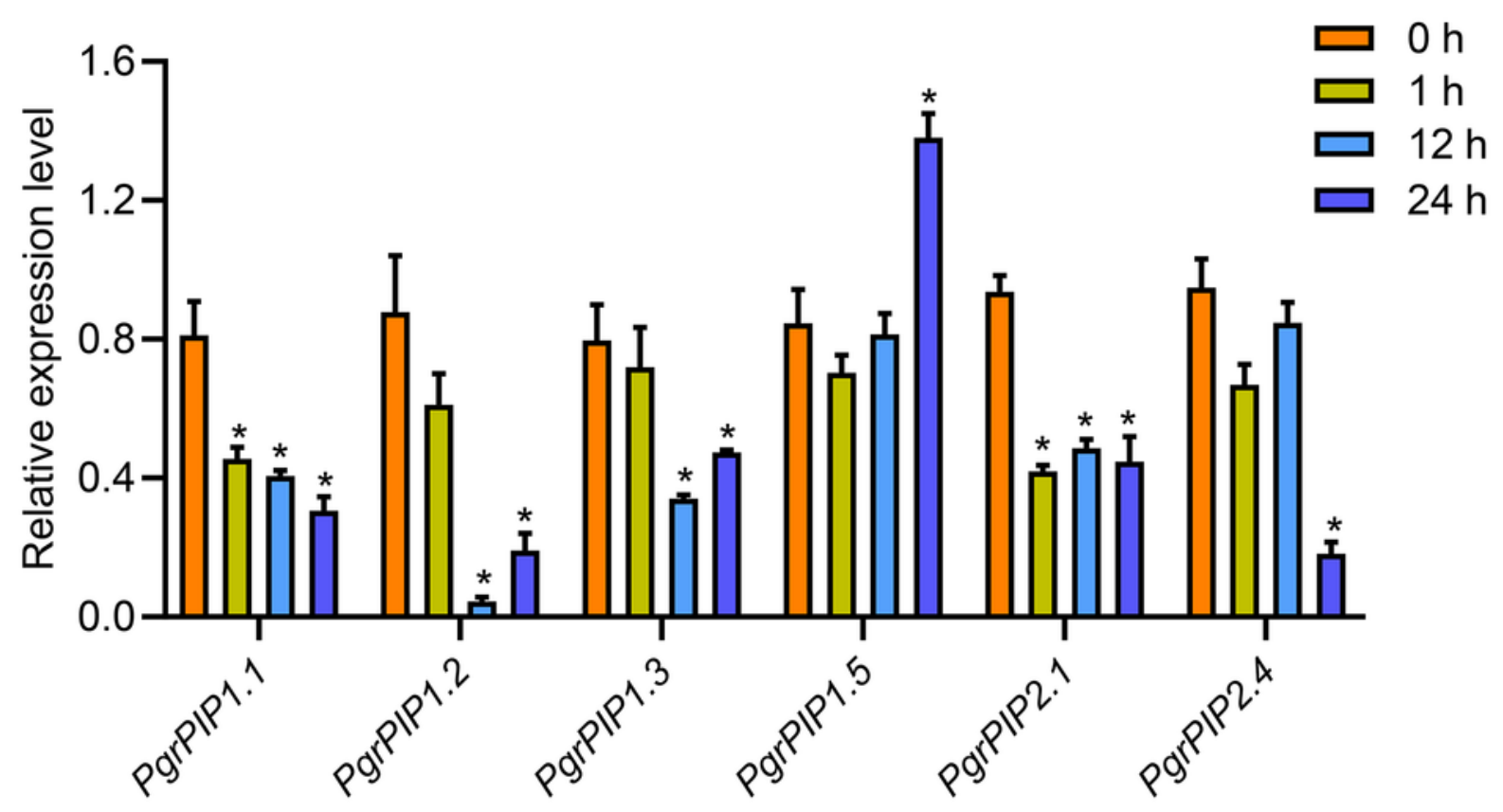




\section{Figure 6}

Water accumulation in seed coats of pomegranate.

The abbreviations in the sample designations represent the cultivar names: $D$ forP.

granatum'Dabenzi' and T forP. granatum 'Tunisia.' The second letter represents tissue: $\mathrm{O}$ and I stands for the outer seed coats and the inner seed coat, respectively. The numbers represent the three stages of seed coat development at different days after flowering (DAF). Different letters indicate a significant difference $(P<0.05)$.

A Dabenzi

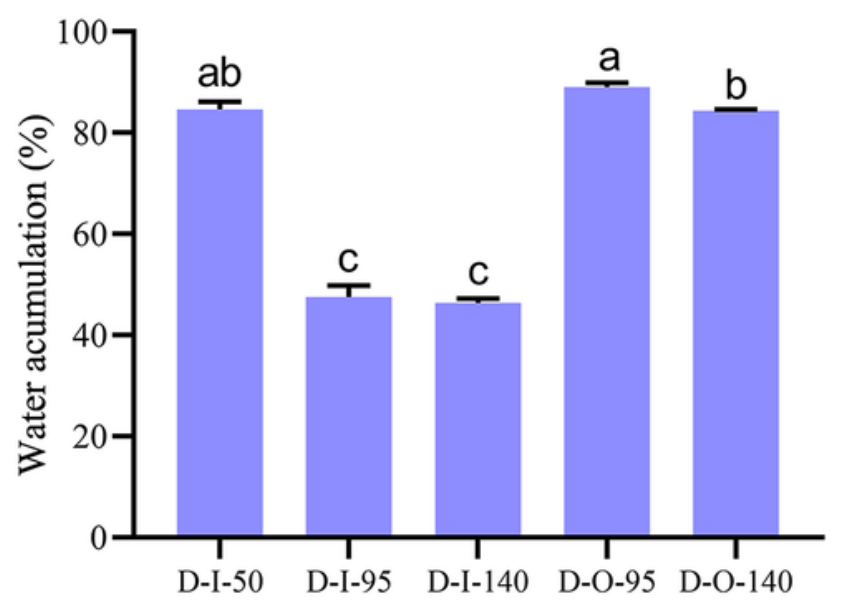

B Tunisia

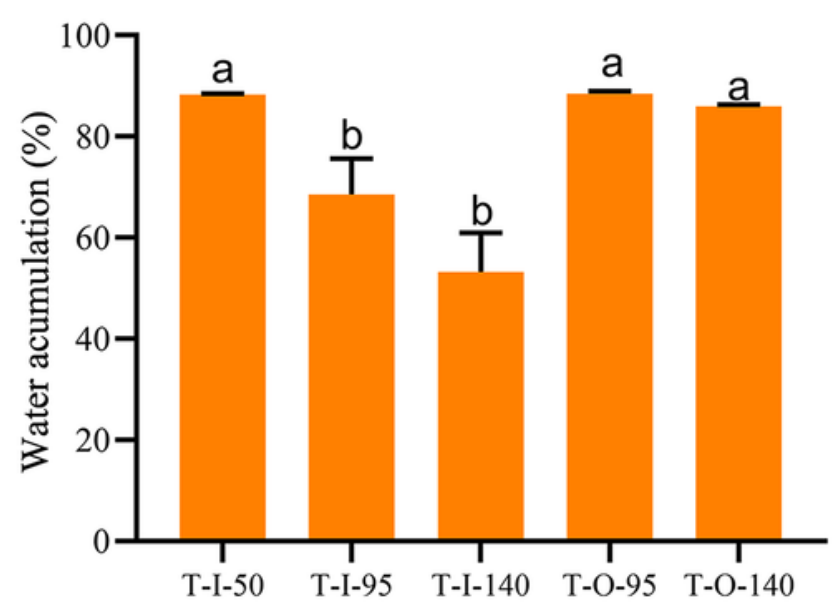


Figure 7

Linear regression between gene expression of PgrAQPs and water accumulation.

Correlation analysis between gene expression of PgrPIP1.3, PgrPIP2.8 and PgrSIP1.2 andwater accumulation in seed coats from P. granatum 'Dabenzi' (A, C, E) and P. granatum'Tunisia' (B, D, F). 
'Dabenzi'

A

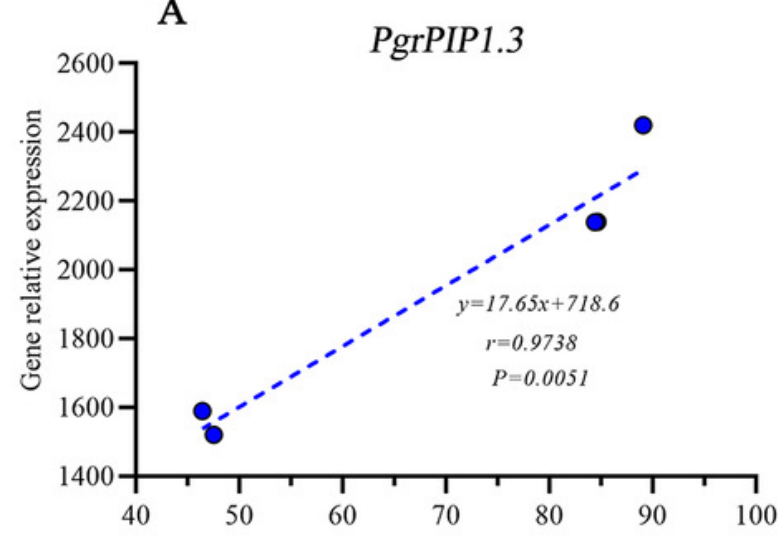

C

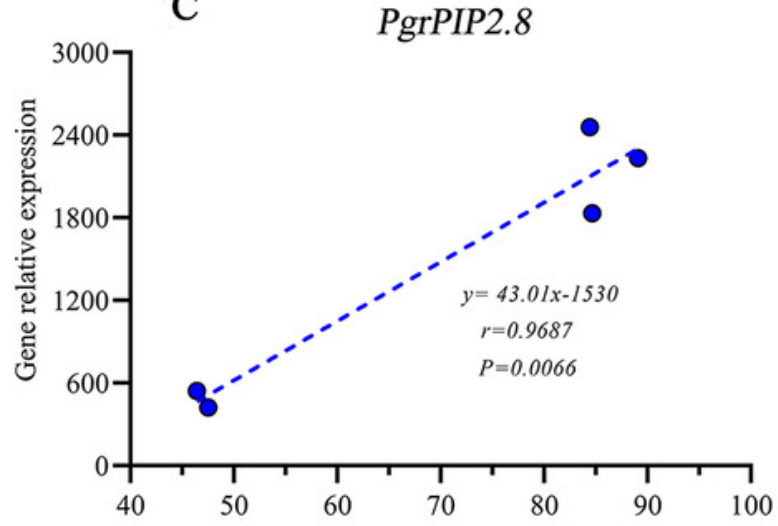

E

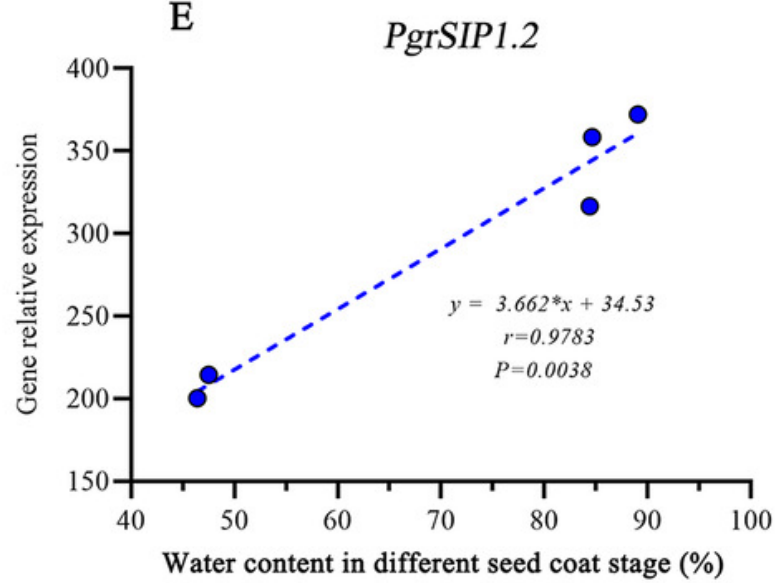

'Tunisia'

B

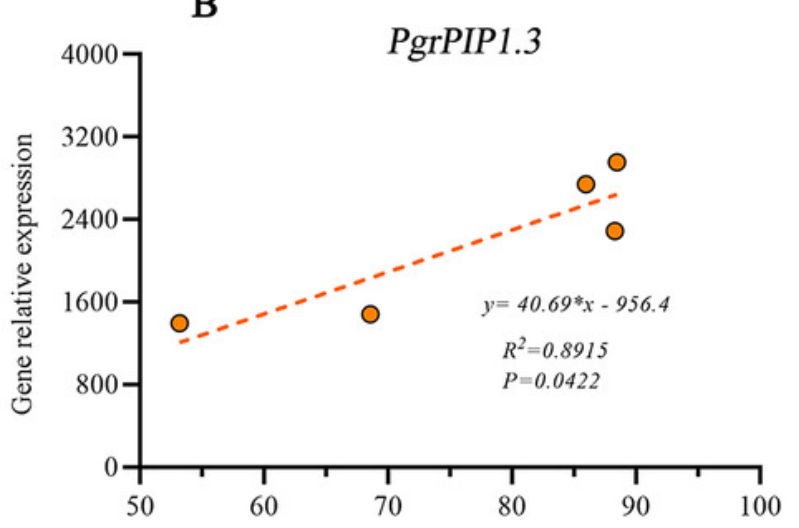

D
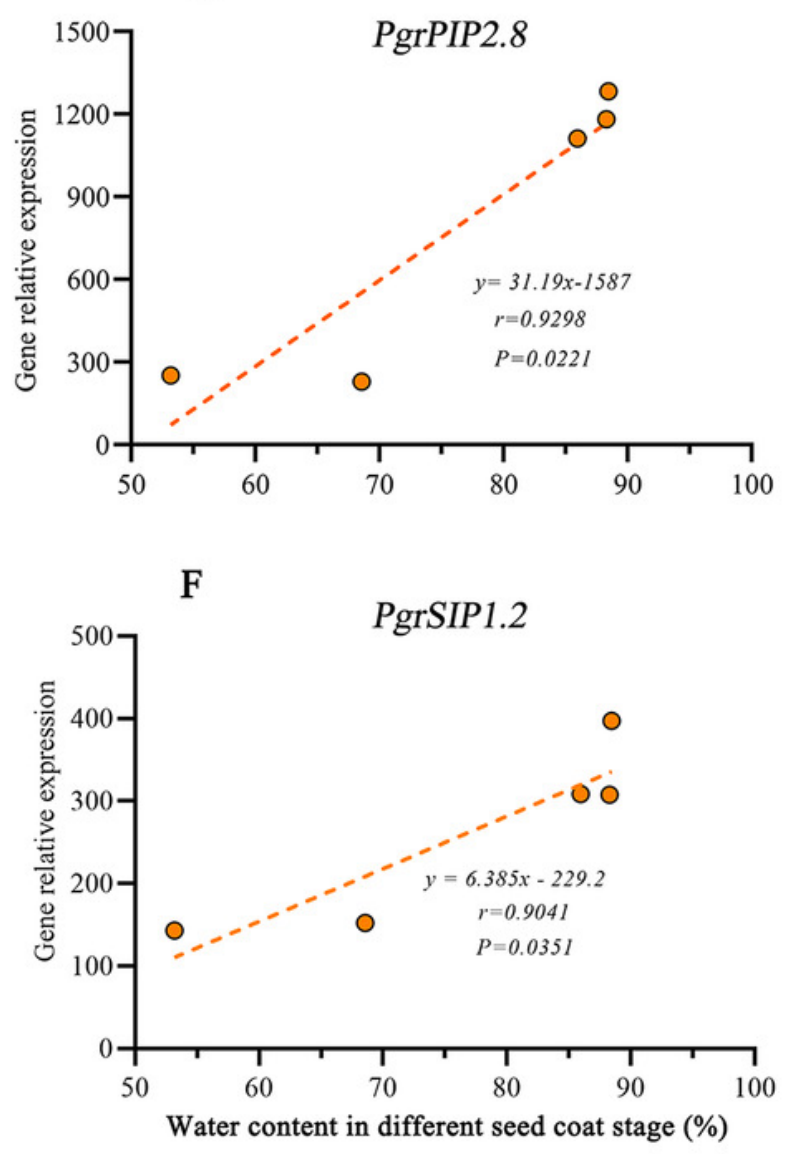


\section{Table $\mathbf{1}$ (on next page)}

The Ka and Ks values of duplicated PgrAQP gene pairs. 
$1 \quad$ Table 1 The $K a$ and $K s$ values of duplicated PgrAQP gene pairs.

\begin{tabular}{|c|c|c|c|c|c|c|c|}
\hline Duplicated gene pairs & $\begin{array}{c}\text { Duplicate } \\
\text { type }\end{array}$ & $K a$ & $K s$ & $K a / K s$ & $\begin{array}{l}\text { Time } \\
\text { (Mya) }\end{array}$ & $\begin{array}{c}\text { Purify } \\
\text { selection }\end{array}$ & $\begin{array}{l}\text { Note: } K a \text {, } \\
\text { non- }\end{array}$ \\
\hline PgrTIP1.2 vs PgrTIP1.5 & Segmental & 0.074 & 1.54 & 0.048 & 5.12 & Yes & synonym \\
\hline PgrTIP3.1 vs PgrTIP3.2 & Segmental & 0.254 & 1.19 & 0.213 & 3.97 & Yes & ous \\
\hline PgrPIP1.5 vs PgrPIP1.1 & Segmental & 0.078 & 0.86 & 0.090 & 2.88 & Yes & substitu \\
\hline PgrPIP2.5 vs PgrPIP2.2 & Segmental & 0.123 & 1.26 & 0.098 & 4.21 & Yes & $K s$ \\
\hline PgrPIP2.7 vs PgrPIP2.3 & Segmental & 0.102 & 1.38 & 0.074 & 4.59 & Yes & synonym \\
\hline PgrPIP2.6 vs PgrPIP2.7 & Segmental & 0.073 & 1.30 & 0.057 & 4.32 & Yes & ous \\
\hline PgrTIP1.2 vs PgrTIP1.1 & Segmental & 0.096 & 2.09 & 0.046 & 6.97 & Yes & substituti \\
\hline PgrTIP1.2 vs PgrTIP1.7 & Tandem & 0.101 & 0.52 & 0.194 & 1.74 & Yes & on \\
\hline
\end{tabular}

14 Million years ago. 